Portland State University

PDXScholar

$1-1-2011$

\title{
3D Interdigitated Electrode Array (IDEA) Biosensor For Detection Of Serum Biomarker
}

Dheeraj Kumar Bhura

Portland State University

Follow this and additional works at: https://pdxscholar.library.pdx.edu/open_access_etds Let us know how access to this document benefits you.

\section{Recommended Citation}

Bhura, Dheeraj Kumar, "3D Interdigitated Electrode Array (IDEA) Biosensor For Detection Of Serum Biomarker" (2011). Dissertations and Theses. Paper 274.

https://doi.org/10.15760/etd.274

This Thesis is brought to you for free and open access. It has been accepted for inclusion in Dissertations and Theses by an authorized administrator of PDXScholar. Please contact us if we can make this document more accessible: pdxscholar@pdx.edu. 
3D Interdigitated Electrode Array (IDEA) Biosensor For Detection Of Serum

Biomarker

\author{
by
}

Dheeraj Kumar Bhura

A thesis submitted in partial fulfillment of the requirements for the degree of

\author{
Master of Science \\ in \\ Electrical and Computer Engineering
}

Thesis Committee:

Raj Solanki, Chair

James Morris

Erik Sànchez

Portland State University

2011 


\section{Abstract}

Miniaturization, integration and intelligence are the developing trends for sensor, especially for biosensors. The development of microelectronics technology is a powerful engine to fulfill this objective. It is well known that the microelectronic fabrication process in proven technology for fabrication of integrated circuits. Advances in the field of micro-electronics and micro-mechanical devices combined with medical science have led to the development of numerous analytical devices in monitoring of a wide range of analytes. The unique properties of nanoscale materials offers excellent prospects for interfacing biological recognition events with electronic signal transduction and for designing a new generation of bio-electronic devices exhibiting novel functions. Biosensor development has the potential to meet the need for rapid, sensitive, and specific detection of pathogenic bacteria from natural sources. This work focuses on development of one such electrochemical biosensor platform and discusses different aspects related to the design of biosensor and biodetection systems. A new transducer for biosensor applications based on 3-dimensional, comb structured interdigitated electrode arrays was chosen mainly for two reasons. Firstly, this geometry allows the monitoring of both resistivity and dielectric constant of solution, thus making interdigitated electrodes more versatile tools than other kind of transducers. Second, they present short electric field penetration depths, which make them more sensitive to changes occurring close to their surface (20 - $100 \mathrm{~nm}$ above the surface). This fact enables the monitoring of local changes in the vicinity of interest. Binding of analyte molecules to the 
chemically modified transducer surface induces important changes in the conductivity between the electrodes. Interdigitated electrodes have been employed to detect the presence of Anti-Transglutaminase ( $\alpha \mathrm{TG}$ ) antibodies, that are established biomarkers for Celiac disease which is due to gluten allergy. The biosensor was optimized for specific and sensitive detection of this biomarker. The sensor showed a sensitivity down to picomolar(pM) concentration of the biomarker. Gold nanoparticles were further used for signal enhancement so as to bring the sensor performance closer to Enzyme linked immunosorbant assay (ELISA). 


\section{Acknowledgments}

First and foremost I offer my sincerest gratitude to my advisor, Dr Raj Solanki, who has supported me throughout my thesis with his patience and knowledge whilst allowing me the room to work in my own way. His perpetual energy and enthusiasm in research had motivated all his advisees, including me. In addition, he was always accessible and willing to help his students with their research. As a result, research life became smooth and rewarding for me. One simply could not wish for a better or friendlier advisor. I would also like to thank my thesis committee members, Dr.James Morris and Dr.Erik Sànchez for their efforts and instruction. I would like to express my deepest gratitude to Dr. Kanwar Vikas Singh, the Post-Doctoral research associate in our group for his invaluable guidance in the biological aspects of the project, the coffee breaks and numerous technical discussions throughout the entire research period. All my lab buddies made it a convivial place to work. My stay at PSU was very entertaining, thanks to friendship of Chris, Rahul and many others. I would also like to acknowledge Dr. David R. Evans, Dr. Andrei Gindilis for their guidance in the project and the entire team from Sharp labs of America for funding this research. I am also grateful to Dr. Jeff King and the group at Virogenomics for their collaborative efforts in this research and suggestions for overcoming several minor problems during the research. Finally, I thank my parents and my brother and sister for their unflagging love and support throughout my life; this thesis is simply impossible without them. 


\section{Contents}

$\begin{array}{ll}\text { Abstract } & \text { i }\end{array}$

Acknowledgments $\quad$ iii

List of Figures $\quad$ vi

1 Introduction $\quad 1$

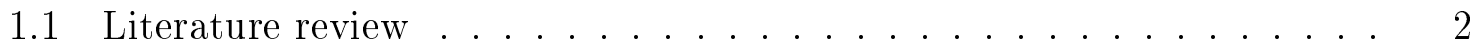

1.1.1 Microelectronics Technology ............. 2

1.2 Biosensor Classification . . . . . . . . . . . . . . 4

1.2.1 Biological Recognition Element (Receptor) . . . . . . . . . 4

1.2.2 Physicochemical signal transduction: . . . . . . . . . . . 6

1.3 Impedimetric biosensors . . . . . . . . . . . . . . . . 13

1.4 Thesis Statement ........................... 14

2 Sensor Fabrication and Characterization $\quad 16$

2.1 Material Selection . . . . . . . . . . . . . . 16

2.2 Device Fabrication . . . . . . . . . . . . . 17

2.3 Device Characterization . . . . . . . . . . . . . 19

2.3.1 Electric Field distribution of IDEA's . . . . . . . . . . . . 19

2.4 Electrochemical Impedance-Theory . . . . . . . . . . . . . 22 
2.5 Electrochemical Impedance Spectroscopy . . . . . . . . . . . . . . . 24

2.5.1 Equivalent circuit Model ............... 24

3 Optimization of 3D IDEA's as Biosensor $\quad 31$

3.1 Device Characterization:Sensitivity to Electrochemical Environment . . 31

3.2 Biofunctionalization of IDEA's . . . . . . . . . . . . . . 34

3.2.1 Surface Modification's . . . . . . . . . . . . 34

3.2.2 Immobilization . . . . . . . . . . . . . 35

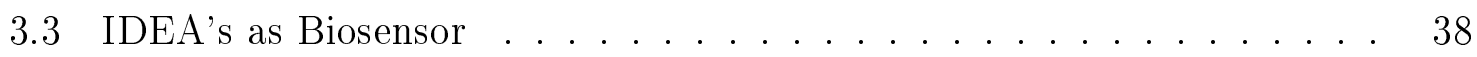

3.3 .1 Sensor Specificity . . . . . . . . . . . . . . 38

3.3.2 Biosensor Vs Bioassay . . . . . . . . . . . . 41

3.4 Biosensor Assay . . . . . . . . . . . . . . . . . 44

3.4.1 Signal Enhancement using Nanoparticle conjugates . . . . . . 46

3.4.2 ELISA vs Biosensor . . . . . . . . . . . . . . 49

4 Summary Conclusions $\quad 53$

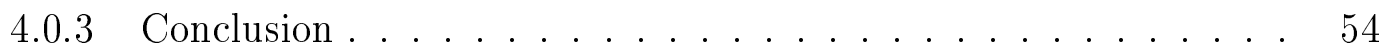

4.0.4 Future work ...................... 54

$\begin{array}{ll}\text { References } & 56\end{array}$ 


\section{List of Figures}

1.1 Biosensor principle-Generic representation showing different elements of

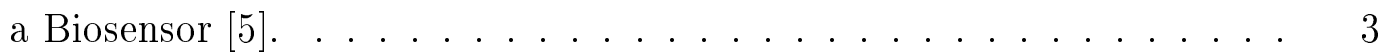

1.2 Ion migration in solution volume and electrolyte conductivity [15] . . 11

2.1 Fabrication Procedure ...................... 17

2.2 (a)SEM image,(b) AFM image,(c) Sensor Chip, (d) Microscopic view of the comb like electrodes structures . . . . . . . . . . 18

2.3 IDE cross section design . . . . . . . . . . . . . . 20

2.4 E-field strength(E) and current density $(\mathrm{J})$ distribution of the electrodes 21

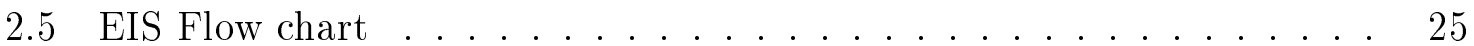

2.6 Randles Circuit of electrochemical cell(R-resistance of electrolyte, $\mathrm{C}_{\mathrm{dl}^{-}}$ double layer capacitance, $\mathrm{R}_{\mathrm{ct}^{-}}$charge transfer resistance, $\mathrm{Z}_{\mathrm{w}}$-warburges resistance) $[10] \ldots \ldots \ldots \ldots \ldots \ldots$

2.7 Equivalent Circuit Model $[24] \ldots \ldots$. . . . . . . . . . 27

2.8 Equivalent Circuit Model with CPE . . . . . . . . . . 28

2.9 Nyquist plots and corresponding equivalent circuits $[18,26] \ldots$. . . . 29

3.1 Niquist plots at different concentration of $K\left[\mathrm{Fe}[\mathrm{CN}]_{6}\right]^{-3 /-4} \ldots \ldots$

3.2 Z-view software template . . . . . . . . . . . 33

3.3 Plot showing $R_{\text {ct }}$ values obtained form Z-view software . . . . . . . 33

3.4 Formation of Self-Assembled Monolayers . . . . . . . . . . . . . . 37 
3.5 Protein Immobilization: (a) Bare Electrode (b) SAM layer (c) Protein immobilized on SAM ...................... 38

3.6 Chip showing two regions with different protein immobilization. . . . . 39

3.7 Specific vs Non-Specific binding. . . . . . . . . . . . . . 39

3.8 Plots showing Specific vs Non-Specific binding. . . . . . . . . . . . 41

3.9 On-chip ELISA, the developed colour increase with decreasing dilution of serum samples indicating the presence of more Abs bound to the

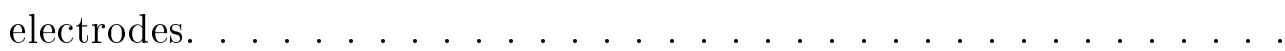

3.10 On-chip ELISA, the optical density at $450 \mathrm{~nm}$ was recorded and plotted against the serum dilutions. Please see text for details. . . . . . . . . 43

3.11 Topology for the experimental procedure. . . . . . . . . . . . . 44

3.12 Nyquist Plots showing the concentration profile of Anti TG serum anti-

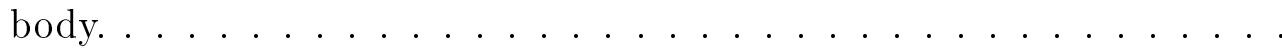

3.13 Plot showing $R_{c t}$ values corresponding to the relative impedance change with antiserum dilutions. . . . . . . . . . . . . 46

3.14 Protein conjugated nanoparticle binding to the electrode surface. . . . . 48

3.15 Nyquist plot with and with out nano particles. . . . . . . . . 49

3.16 Plot showing the comparison of the $\mathrm{R}_{\mathrm{ct}}$ values with and with out nanopar-

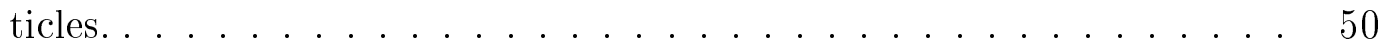

3.17 Fitting curves of Pr-NP data, here 1, 2, 3 are 1 Million, $10 \mathrm{~K}, 100$ dilutions of the protein conjugated with NPs and 1', 2', 3' are the fit curves obtained from Z-view analysis. . . . . . . . . . . 51

3.18 Histogram comparing three different detection methods. . . . . . . . 51

3.19 Plot showing the normalized signal strength of label free, Protein conjugated NPs, ELISA. . . . . . . . . . . . . . 52 


\section{Chapter 1}

\section{Introduction}

Diagnosis and monitoring of various diseases necessitate intensive efforts for routine examination of blood samples and other associated tests. These however require typical analytical techniques, need efficient hands to perform the job and time for collecting the desired samples for performing clinical tests. Hence there is an urgent need in medical diagnostics for readily usable, accurate, fast and inexpensive devices. Reliable and accurate information on the desired biochemical parameter is an essential prerequisite for effective healthcare management. Technological advances in the past few decades have led to the development of numerous analytical devices for monitoring a wide range of analytes. In this context biosensors are considered to provide a viable solution to the problem posed by the contemporary healthcare industry. The desire to measure and understand every single variable in our environment has been an impetus behind the growth of these innovative tools. Biosensors are one group of analytical devices that has attracted considerable attention in recent times [1]. This is because the biosensing devices offer considerable advantages, such as small size, faster response and low cost. A fundamental challenge pertinent to biosensor design lies in the molecular understanding of the relationship between surface and reactivity. Understanding the fundamental process that governs the biosensor response in most cases leads to the development of an electro analytical device with superior selectivity, excellent chemical stability, higher 
sensitivity, and lower detection limit [2].

\subsection{Literature review}

\subsubsection{Microelectronics Technology}

Micro technology involves not only the possibility of shrinking the dimensions of the mechanical, optical and micro fluidic functionalities down to the scale of microelectronics circuits, but it also opens new industrial applications in various fields. Today the most promising microsystems are the result of modular rather than integrated design [3]. The microsystems incorporates several interdisciplinary technologies, materials and components all in a single system. Microsystem technology is rapidly becoming a key element in the development of advanced instrumentation for analytical research [4]. Micro technology or Microsystemtechnology is commonly regarded as a general strategy of success, not only in electronic components and systems but also for a wide variety of mechanical, optical, acoustic, thermal, fluidic, and chemical or biochemical functional units [3]. The application of microfabrication techniques to develop micro biosensors has added a new impetus to this endeavor, especially in commercializing biosensors for routine tests and in vivo monitoring in biological and medical research. The bio-sensor manufactured by employing the microfabrication technologies exhibit

many advantages as follows: (a) the mass-production of sensors with good reproducibility, leading to decreased production costs as well as lowering power consumption; (b) the integration of sensors and IC resulting in compact size and higher performance; (c) the low cost and compact size is beneficial to both in vivo and in vitro analysis in medical diagnosis. These micro-sized electrodes display a number of desirable properties, including high current density due to non-planar diffusional contributions to the net Faradic current, very short time constants, and low ohmic drop. These features offer 
properties such as wider range of resistive solutions and very high scan rates. Therefore, use of microtechnology for realization of complex instruments offer a reduction in the required resources and improved performance. This work focuses on one such microelectronic electrochemical biosensor system.

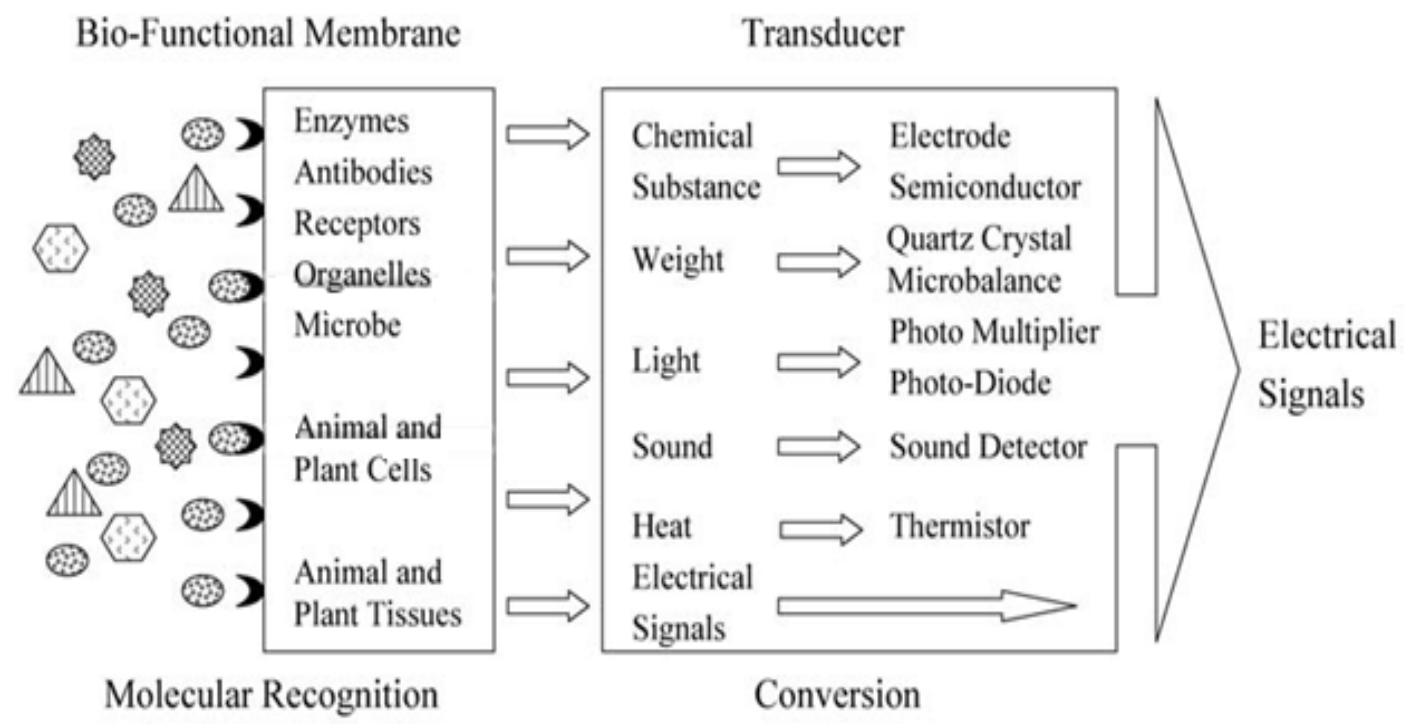

Figure 1.1: Biosensor principle-Generic representation showing different elements of a Biosensor [5].

A sensor is a device that converts an observed change (physical or chemical) into a measurable signal. A bio-sensor can be generally defined as a device that consists of a biological recognition system, often called a bioreceptor, and a transducer. The interaction of the analyte with the bioreceptor is designed to produce an effect measured by the transducer, which converts the information into a measureable unit, such as an electrical signal [6]. The analyte that this sensor detects and measures may be purely chemical (environmental pollutants), although biomolecules (disease biomarkers) can also be the target analyte. The key difference is that the recognition element is biological in nature. Electrochemical Biosensors can essentially serve as low cost and highly efficient devices for detection of a disease biomarker. The common feature 
binding all the electrochemical sensors is that they rely on the detection of a electrical property i.e, (potential, resistance and current) and accordingly they are classified as potentiometric, conductometric and amperometric senors respectively [7].

\subsection{Biosensor Classification}

Biosensors may be classified accordingly to the biological specificity-conferring mechanism or alternatively the mode of physicochemical signal transduction. The biological recognition may be based on a chemical reaction catalyzed by or an equilibrium reaction with, biomolecules that have been isolated, engineered or present in their original biological environment. In the latter case, equilibrium is generally reached and there are no further, if any, net consumptions of analyte by immobilized complexing agents incorporated into the sensor [7].

\subsubsection{Biological Recognition Element (Receptor)}

The biological recognition element or bioreceptor is a crucial component of the biosensor device. These elements are highly selective and sensitive to the analytes when combined with electrochemical, optical, piezoelectric, magnetic, thermometric transducers. Generally, one can draw two principal classes of biosensors that are distinguished from one another by the nature of the processes and by the involved biochemical or biological components [8].

\section{Bio-Catalytic}

A biosensor with bio-catalytic recognition element is based on a reaction catalyzed by micro molecules (i.e, Enzymes, microbes, plant and animal cells, plant and animal tissue), which are present in their original biological environment, have been isolated 
previously or have been manufactured. Thus, a continuous consumption of substrates is achieved by immobilized biocatalyst incorporated into the sensor: transient or steady state response is monitored by the integrated detector [8]. Off all enzyme recognition element based biosensors, the glucose biosensor based on glucose oxidase is the most widely studied and acclaimed bio-catalytic sensor. Unlike bio-catalytic sensors, the focus of this work is on bio-affinity sensors [6,9].

\section{Bio-affinity (Immunological or hybridization)}

A Bio-Affinity sensor is based on the interaction of the analyte with the macromolecules or molecular assemblies that have been established on to the transducer surface. Biosensors using biocomplexing receptors are based on immunochemical reactions, i.e binding of antigen $(\mathrm{Ag})$ to a specific antibody $(\mathrm{Ab})$, taking place on the transducer surface which can be directly (Heterogeneous phase immunosensor) or indirectly (Homogeneous phase immunosensor) detected. In the homogeneous phase approach, the detection of the immune- complex is achieved by labeling the antibody or antigen depending on the immunoassay format (Sandwich type, competition, capture), performing optical detection. Heterogeneous phase approaches are label free methods, in which the specific binding events between the antibody and the target analyte (the antigen) is monitored by the change or variation in physicochemical properties. In this case, the detection methods include Electrochemical Impedance Spectroscopy (EIS) and Surface Plasmon resonance (SPR). In addition, these direct detection methods can also provide kinetics information on antigen-antibody interactions. In affinity based biosensing the detection of a bio-molecule is a two step process (1) binding the desired target while excluding non target binding (this is affinity step) and (2) detecting a change in the surface properties (the readout step). The affinity step is based on the surface chemistry and biological binding, while the readout step is based on the physics 
of detection plus all the associated signal processing [6,9,10]. Affinity biosensors are further classified based on the transduction technology exercised.

\subsubsection{Physicochemical signal transduction:}

Transduction may be achieved via a great variety of methods. Most of the biosensingrelated transduction can be categorized into one of the three main classifications: (1) Electrochemical Transduction (2) Optical Transduction and (3) Mechanical Transduction [8].

\section{Optical Transduction}

The modulation in optical properties such as UV-vis absorption, bio- and chemiluminescence, reflectance, diffraction and fluorescence brought by the interaction of the biocatalyst with the target analyte is the basis for optical biosensors $[1-4,11]$. Optical biosensors offer advantage of compactness, flexibility, resistance to electrical noise, and a small probe size. The interactions between the electromagnetic radiation and the compositions labels bonded to the immunoreagents allow detection of affinity reactions via, variations in fluorescence, absorbance or polarization [12]. In optical diffraction based devices, a silicon wafer is immobilized with protein via covalent bonding. The wafer is then exposed to UV radiation through a photo mask making the antibodies inactive in the exposed regions. When the wafer is incubated in an analyte solution, antibody-antigen bindings occur in the active regions, thus creating a diffraction grating. This grating produces a diffraction signal when illuminated with a light source such as laser. The resulting signals can be measured or further amplified before measuring for improved sensitivity. Insensitivity to electric interfaces and high safety for in vivo studies are the attractive features of these kind of sensors however when compared to other immunosensors they are less advantageous because of the time consumed in 
analysis and lack of detection selectivity due to nonspecific adsorption and high cost of instrumentation $[6,8]$.

\section{Mechanical Transduction}

Generally mechanical bio(chemical) sensors are referred to as "mass sensitive" due to gravity or thickness measurements of thin rigid films in vacuum or gaseous environment. Acoustic wave devices give information on two physical quantities, the acoustic energy storage and the energy dissipation which can be respectively measured by the

frequency shift and the damping of the acoustic oscillation due to mass changes [8]. A piezoelectric immunosensor is a device based on materials such as quartz crystals, which resonate on application of an external alternating electric field. The frequency of the resulting oscillation is a function of the mass of the crystal. Thus, interaction of an analyte in a sample with the corresponding antibody, previously immobilized by passive adsorption or by covalent interaction to a quartz crystal, will increase the overall mass, which is measured as a change in the frequency of oscillation. The two types of piezoelectric immunosensors are bulk acoustic (BA) devices and surface acoustic wave (SAW) devices. In the BA device, the specific adsorption of an analyte with the corresponding antibody-immobilized crystal occurs at the surface, which is connected to an oscillating circuit, but resonance occurs on the entire mass of the crystal. Thus, the increase in mass will result in a decrease in the resonant frequency, which is a function of the analyte concentration. In the SAW immunosensor device, an acoustic wave travels only over the surface of the crystal that is coated with a specific antibody. A change in the frequency will occur as the analyte in a sample binds to the corresponding immobilized antibody [8]. 


\section{Electrochemical Transduction}

Electrochemical transducers have a very important role in healthcare because of the advantage of their technology, which include low cost, portability and the possibility of directly identifying and quantifying specific compounds in complex samples. There are three types of electrochemical transducers: Amperometric, potentiometric, Impedimetric/conductometric. Among electrochemical biosensors, the oldest ones, which have led to the higher number of ready to use devices, are based on the monitoring of electron transfer processes, thus belonging to amperometric category [13]. Amperometric biosensors operate at a constant potential, at Pt-, Au- or C- based working electrode or an array of electrodes, with respect to a reference electrode and involves the detection of the current generated by the oxidation or reduction of species at the surface of the electrode. The generated current is directly correlated to the bulk concentration of the electroactive species or its production or consumption rate within the adjacent biocatalytic layer. As biocatalytic reactions are considered to be first-order dependent on the bulk analyte concentration, such study-state currents are proportional to the bulk analyte concentration [7]. Conventional potentiometric biosensors consist of an ion-selective electrode ( $\mathrm{pH}$, ammonium, chloride and etc) or a gas-sensing electrode $\left(\mathrm{pCO}_{2}\right.$ and $\left.\mathrm{pNH}_{3}\right)$ coated with immobilized microbe layer. Microbe consuming analyte generates a change in the potential resulting from an ion accumulation or depletion. The basic principle behind potentiometric transducers is the development of significant potential at the electrode by accumulation of charge and hence increased charge density at an electrode surface. The measured potential difference developed between the working electrode and the reference electrode is correlated to the analyte activity $a_{i}$ in the sample through Nernst Relation 


$$
\mathbf{E}=\mathbf{E}_{\mathbf{0}}+(\mathbf{R T} / \mathbf{n F}) \ln \mathbf{a}_{\mathbf{i}},
$$

where $E_{0}$ is standard potential for $a_{i}=1 \mathrm{~mol} \mathrm{l}^{-1}[14], R$ is gas constant, $F$ is the faraday constant, $\mathrm{T}$ is the temperature in $\mathrm{K}$ and $\mathrm{n}$ is the total number of charges on ion i. Due to the logarithmic relationship between a potential generated and analyte concentrations, a wide detection range is possible. However, this method requires a very stable reference electrode, which may be a limitation of the transducers [14].

Conductometric sensing is a promising modality for electrochemical sensors due to relative simplicity of the measurement, and ease with which miniature sensors can be fabricated. These biosensors are based on the fact that almost all enzymatic reactions involve either consumption or production of charged species and therefore, lead to a global change in the ionic composition or the impedance of the tested sample between the electrodes. Current work is focused on conductometric affinity biosensors using interdigitated electrodes as transducers. Biosensors based on conductometric principles exhibit various advantages such as

- Large scale production and miniaturization of the electrodes is possible using inexpensive technology.

- Reference electrode is not required and the transducers are not light sensitive.

- Power consumption is less because the driving voltages are significantly low.

- Large spectrum of compounds or different nature can be determined on the basis of various reactions and mechanisms.

These types of devices monitor either impedance $(Z)$ or its components resistance $(R)$, capacitance (C), Inductance typically has minimal influence in a typical electrochemical setup [6,15]. Thus the expression of impedance is as follows 


$$
Z^{2}=R^{2}+1 /(2 \Pi f C)^{2},
$$

where $\mathrm{f}$ is the frequency. Conductance is inverse of resistance and vice versa, so these type of sensors are reffered to as Impedimetric. Alternating voltage amplitudes ranging from a few to $100 \mathrm{mV}$ are applied to the two electrodes of the impedance biosensor. In recent years there have been some promising approaches in hybridization of DNA fragments previously amplified by a polymerase chain reaction that has now been monitored by an impedance biosensor. A model impedance immunosensor containing electro deposited polypyrrole film with captured avidin connected through biotin to anti-human IgG was able to detect antibodies at concentrations as low as $10 \mathrm{pg} / \mathrm{ml}$.

\section{Working Principle}

Conductometric biosensors can be considered as generic sensor because many biocatalytic reactions in a solution produce or consume ionic species thus resulting in conductivity changes.

One of the most important characteristic of electrolytic solutions is their capability to carry electric current. Compounds with strong polar bonding dissociate in solvents with a high dielectric constant resulting in positively charged anions and negatively charged cations. The insertion of two similar metal electrodes in such a solution with a potential applied across them causes migration of the ions to the oppositely charged electrodes, at which the ions will be discharged, a processes which is called electrolysis.

The ion flux, i.e. the number of ions passing through a unit of electrolyte crosssection per unit of time $\left(\mathrm{p}_{\mathrm{i}}\right)$ can be determined by the formula: 


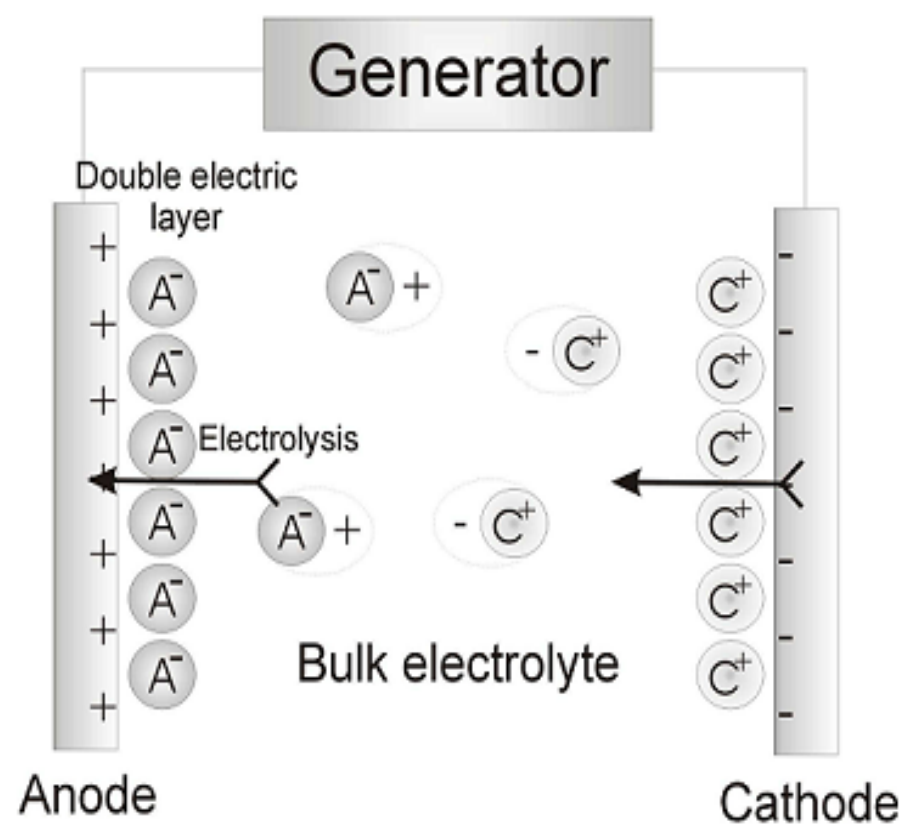

Figure 1.2: Ion migration in solution volume and electrolyte conductivity [15]

$$
\mathbf{p}_{\mathbf{i}}=\mathbf{c}_{\mathbf{i}} \mathbf{v}-\mathbf{k}_{\mathbf{i}} \mathbf{c}_{\mathbf{i}} \operatorname{grad} \mu_{\mathbf{i}}-\mathbf{z}_{\mathbf{i}} \mathbf{v}_{\mathbf{i}} \mathbf{c}_{\mathbf{i}} \operatorname{Fgrad} \psi
$$

where $\mathbf{v}$ - speed of solution flow due to natural or forced convection; $\mathbf{c}_{\mathbf{i}}$ - ion concentration; $\mathbf{k}_{\mathbf{i}}$ - diffusion coefficient; $\mathbf{z}_{\mathrm{i}}$ - charge number; $\mathbf{v}_{\mathrm{i}}$ - speed of ion movement caused by an applied field; F - Faraday number; $\psi$ electrical potential; $\mu$ electrochemical potential [15]. Thus, the first member in formula corresponds to the contribution of the convectional flow of ions at a concentration of $\mathbf{c}_{i}$; the second - the contribution of their molecular diffusion; the third - that of the ion migration induced by the applied potential. Temperature is assumed to be constant $(\mathrm{T}=$ const, grad $\mathrm{T}=0)$ so the thermal diffusion of the ions can be ignored. If only ion immigration caused by the electric field is considered, in case of homogeneous immobile electrolyte, we can ignore the first two 
members of the above equation, then we have

$$
\mathbf{p}_{\mathbf{i}}=-\mathbf{z}_{\mathbf{i}} \mathbf{v}_{\mathbf{i}} \mathbf{c}_{\mathbf{i}} \operatorname{Fgrad} \psi=-\mathbf{c}_{\mathbf{i}} \mathbf{u}_{\mathbf{i}} \operatorname{grad} \psi
$$

the current density, i.e. the current per unit of the system cross-section is an algebraic sum of products of ion fluxes and ion charges:

$$
\mathbf{j}=\mathbf{F} \Sigma \mathbf{z}_{\mathbf{i}} \mathbf{p}_{\mathbf{i}}=\operatorname{Fgrad} \psi \mathbf{z}_{\mathbf{i}} \mathbf{c}_{\mathbf{i}} \mathbf{u}_{\mathbf{i}}
$$

according to Ohms law,

$$
\mathbf{j}=\operatorname{Sgrad} \psi
$$

where S - conductivity, reciprocal of resistance Hence from (1.3) and (1.4).

$$
\mathrm{S}=\mathrm{F} \Sigma \mathbf{z}_{\mathrm{i}} \mathbf{c}_{\mathbf{i}} \mathbf{u}_{\mathrm{i}}
$$

Thus, the conductivity of the electrolyte solution depends on the ion concentration and mobility.The resistance of the electrolyte solution is well-known to be in direct proportion to the distance "L" between the immersed electrodes and reciprocal to their area A, therefore:

$$
\mathbf{S}=\chi(\mathbf{A} / \mathbf{L})
$$

where $\chi$ - specific conductivity. This leads to the following conclusions:

- conductometric measurement commonly consists of determining the conductivity of a solution between two parallel electrodes, its value is a sum of all the ions within the solution tested [15]. 
- The immobilization of biomaterials, e.g., enzymes, antibody/antigens or DNA on electrode surfaces alters the capacitance and interfacial electron transfer resistance of the conductive or semiconductive electrodes, which allows the monitoring of the biomaterial interactions.

Since pioneering works of Newman and Martelet on the concept of capacitive, or impedimetric based immunosensors, a lot of work has been done in this specific area. Since this work focuses on the impedance measurement of the biological activity occurring at the electrode/solution interface a brief review of Biosensor is called for as under.

\subsection{Impedimetric biosensors}

Impedance biosensor measures the electrical impedance of an interface in $\mathrm{AC}$ steady state with constant DC bias conditions. This is accomplished by imposing a small sinusoidal voltage at a particular frequency and measuring the resulting current. The resulting current sine wave differs in time(phase shift) with respect to the perturbation(voltage) wave, and the ratio $V(t) / I(t)$ is defined as the impedance. The impedimetric biosensor enables qualitative and quantitative monitoring of target analyte with a biologically functionalized detection layer incorporated on the electrode surface by measuring the changes in the electrical impedance. Further more, the build-up of the sensing biomaterial film on the conductive or semiconductive electrodes alter the capacitance and resistance properties of the electrode-electrolyte interface. Impedance biosensors have been widely adapted as an analysis tool for the study of various biological reactions because of their high sensitivity and reagent less operation. Impedance spectroscopy is a powerfull tool for the analysis of interfacial properties changes of modified electrodes up on biorecognition events occurring at the 
modified surfaces. Impedance measurements provide detailed information on capacitive/resistance changes occurring at the surfaces. Thus Electrochemical Impedance Spectroscopy is becoming an attractive tool to characterize biomaterial films associated with electronic elements, thus, allowing transduction of biorecognition events at the respective surfaces [16].

Impedimetric transducer is a miniature two electrode device designed to measure the conductivity of the thin electrolyte layer adjacent to the electrode surface. However based on the review documents the best design for the development of impedimetric electrodes is an Interdigitated electrode structure [16,17]. Interdigitated electrodes made up of two individually addressable interdigitated comb-like electrode structures that have frequently been suggested as ultra sensitive electrochemical biosensors. Since the signal enhancement effects due to cycling of the reduced and oxidized species are strongly dependent on the inter electrode distances, the nature of the enhancement is due to overlying diffusion layers. Interdigitated electrodes with an electrode separation of less than one micrometer are desired for maximum signal amplification. Fabrication of sub micron structures can only be made by advanced lithography techniques [18].

\subsection{Thesis Statement}

- This research focuses on the development and characterization of an on-chip biosensor platform to detect affinity based bimolecular interactions. The study is aimed to investigate several different aspects of biosensor development as listed below.

- To investigate Micro-scaled gold interdigitated electrode arrays(IDEA) for Transduction of bimolecular interactions into electrical signals.

- Electrochemical Impedance spectroscopy as the detection technique to measure 
changes in electrical impedance of surface modified electrodes, resulting from Antibody(Ab)-Antigen(Ag) affinity interactions .

- To develop an equivalent circuit model to understand the several components of the measured overall impedance of IDE and determine the component that represents the actual measure of signal proportional to the biomolecular interactions at the electrode surface.

- To study different surface modification techniques to increase the sensitivity of the sensor and compare several blocking agents on biofunctionalized gold IDEA to reduce non-specific binding and analyze using EIS.

- To specifically demonstrate that the biosensors realized can detect the presence of anti-Transglutaminaze (anti $\alpha$-Tg) Ab's that are suitable biomarkers for diagnosis of Celiac sprue(Gluten allergy) in blood serum dilutions made in Phosphate Buffered Saline(PBS).

- To compare the sensitivity of the electrochemical impedimetric biosensor with an onchip ELISA, that is currently the gold standard for detection of Ag-Ab affinity interactions.

- To investigate the use of Gold Nanoparticle labels as secondary reagents to increase the sensitivity of the IDEA sensor. 


\section{Chapter 2}

\section{Sensor Fabrication and Characterization}

\subsection{Material Selection}

The electrode material is critical for the sensitivity and selectivity of the impedance system. IDE's used for impedance detection can be fabricated using gold (Au), platinum $(\mathrm{Pt})$, titanium $(\mathrm{Ti})$, chromium $(\mathrm{Cr})$, carbon $(\mathrm{C})$, and indium tin oxide (ITO). They are fabricated on a variety of base materials, but the most commonly used ones are silicon, quartz/glass, and aluminum oxide. Different shapes of the electrodes are used such as planar interdigitated electrodes, octagonal interdigitated ring electrodes, multi-islands of interdigitated electrodes, ring array electrodes, and 3-D micromesh electrodes. The selection of materials for electrode fabrication depends on the intended application, ionic species involved, inertness of the material to the environment, and their suitability to the fabrication process.Electrode materials such as carbon has wider potential window and larger over-potential for water as compared to noble metal electrodes.This feature is expected to improve the sensitivity of electrochemical reactions for redoxactive biological molecules because their reactions are slow on metal electrodes [19]. To obtain a high sensitivity for electrochemical detection of affinity based biomolecule interactions, electrodes with a highly conductive metal like gold is selected to provide continuous redox cycling and is also suitable for microfabrication processes. 


\subsection{Device Fabrication}

The devices bearing Interdigitated Electrode Array structures were fabricated on 6" silicon wafers. First the wafers were cleaned and oxidized thermally by wet oxidation at $950^{\circ} \mathrm{c}$ to give a good quality $1000 \mathrm{~nm}$ thick silicon dioxide layer. In the next step $15 \mathrm{~nm}$ thick layer of $\mathrm{Cr}$ is deposited which acts as adhesive layer to the $200 \mathrm{~nm}$ gold metal on top of it. This was followed by deposition of a layer of TiN which acted as a hard mask that was followed by a photo resist layer. The first photo lithographic step defines pads, connectors and digits of two electrodes by UV exposure through the photo mask layer on to the soft photoresist.

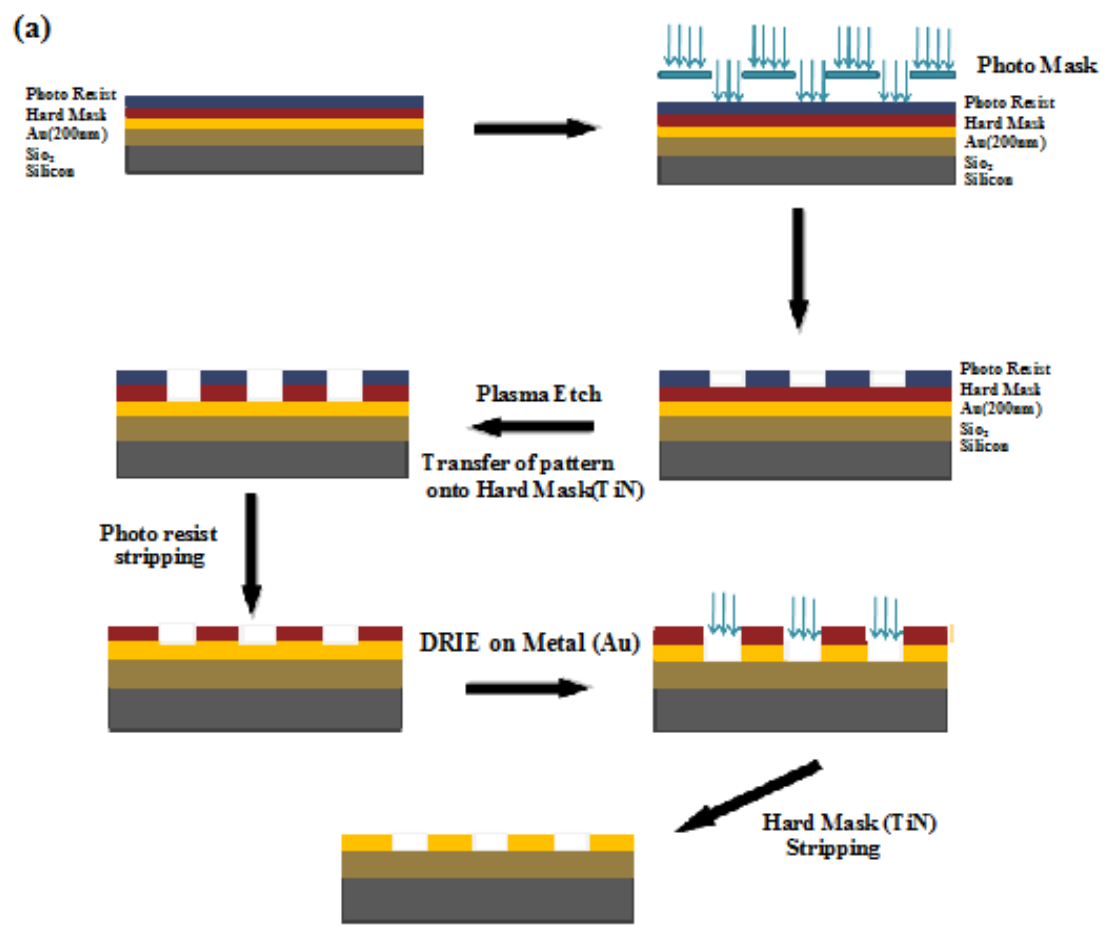

Figure 2.1: Fabrication Procedure

The pattern was developed in the soft mask and transfered on to the hard mask using standard chemical etching followed by the stripping of the soft photo resist. The 
patterning of gold IDEs was done by deep reactive ion etching (DRIE) process that produces IDEs with nearly vertical walls. This was followed by stripping of the TiN hard mask layer. The final process results in a symmetrical interdigitated electrode array with 39 digits from each side with $1500 \mathrm{~nm}$ width separated by a $500 \mathrm{~nm}$ gap between the adjacent electrodes. The total length between the electrodes was 180 micrometer and the height was approximately $200 \mathrm{~nm}$. fig (2.1) shows a detailed sketch of the fabrication processes steps and fig $2.2(\mathrm{a}, \mathrm{b}, \mathrm{d})$ shows the SEM, AFM and optical microscopic images of the fabricated electrodes. There were eight IDE array devices laid on a single chip which resembles a secure digital (SD) card shown in fig 2.2(c). The chip is designed such that all the eight IDE arrays have individually addressable contact points and are designed such that they can be divided into two sets.

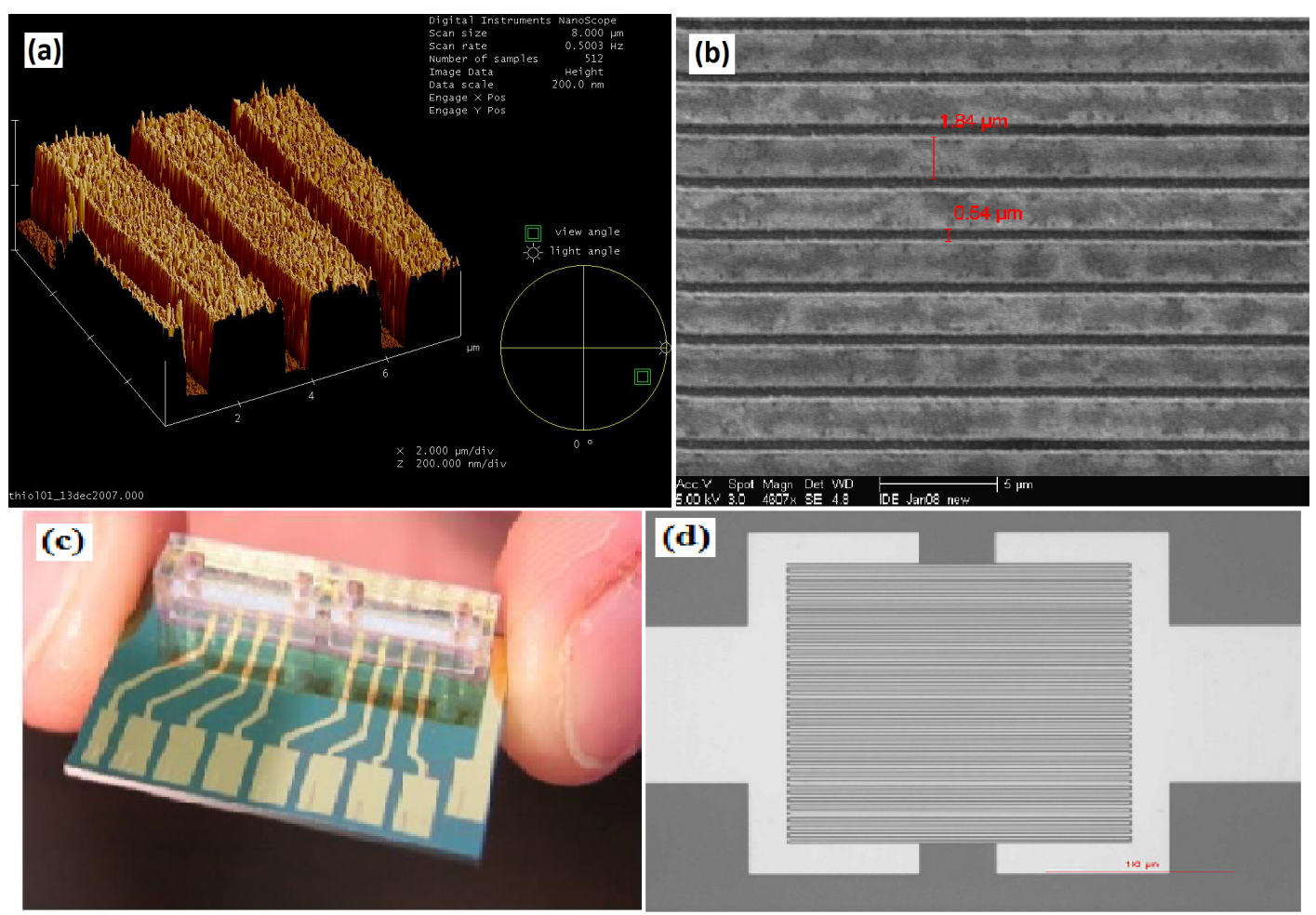

Figure 2.2: (a)SEM image,(b) AFM image,(c) Sensor Chip, (d) Microscopic view of the comb like electrodes structures 


\section{Plexi glass channel fabrication}

A plexi glass channel was custom made with inlet and outlet holes drilled to flow a liquid through the channel. A PDMA gasket was also designed such that it sit on top of the chip surrounding the IDE arrays creating a channel to hold the solution. Plexiglass channel was mechanically held on top of the PDMS stamp so that the solution stays within the channel enclosing all the eight IDEA's on a single chip.This channel holds up to 70 micro liters of solution.

\subsection{Device Characterization}

\subsubsection{Electric Field distribution of IDEA's}

Sensors with nano scaled electrode arrays have an improved sensitivity compared with other impedimetric measurement principles. When antibodies bind to antigens or DNA binds to probes, only a very small region above the surface of the electrode (in the range of few tens to few hundred nanometers) is modified. These electrodes only probe in the region close to the surface and thus show an improved sensitivity. This section reviews the Finite Element Analysis (FEA) analysis performed on the high IDE structure to determine the distribution of the electric field and current density in the vicinity of the IDE. Commercially available FEA tool (Maxwell's Ansoft) has been used. A two dimensional cross section of two pairs of electrodes were considered that an infinite array with specifications as follows, width of the electrode is $1500 \mathrm{~nm}$, height of the electrodes is $200 \mathrm{~nm}$, spacing between the electrodes is $500 \mathrm{~nm}$, oxide thickness is $1 \mathrm{~nm}$ as shown in fig 2.3. A small AC voltage was applied to the electrode pairs, thus different concentrations of cells and biomolecules bound to the surface yielded different electrical impedance changes between the electrode structures. The background medium was considered to be PBS solution with conductivity of $1.45 \mathrm{~S} / \mathrm{m}$ 
and relative permitivity of 80 .

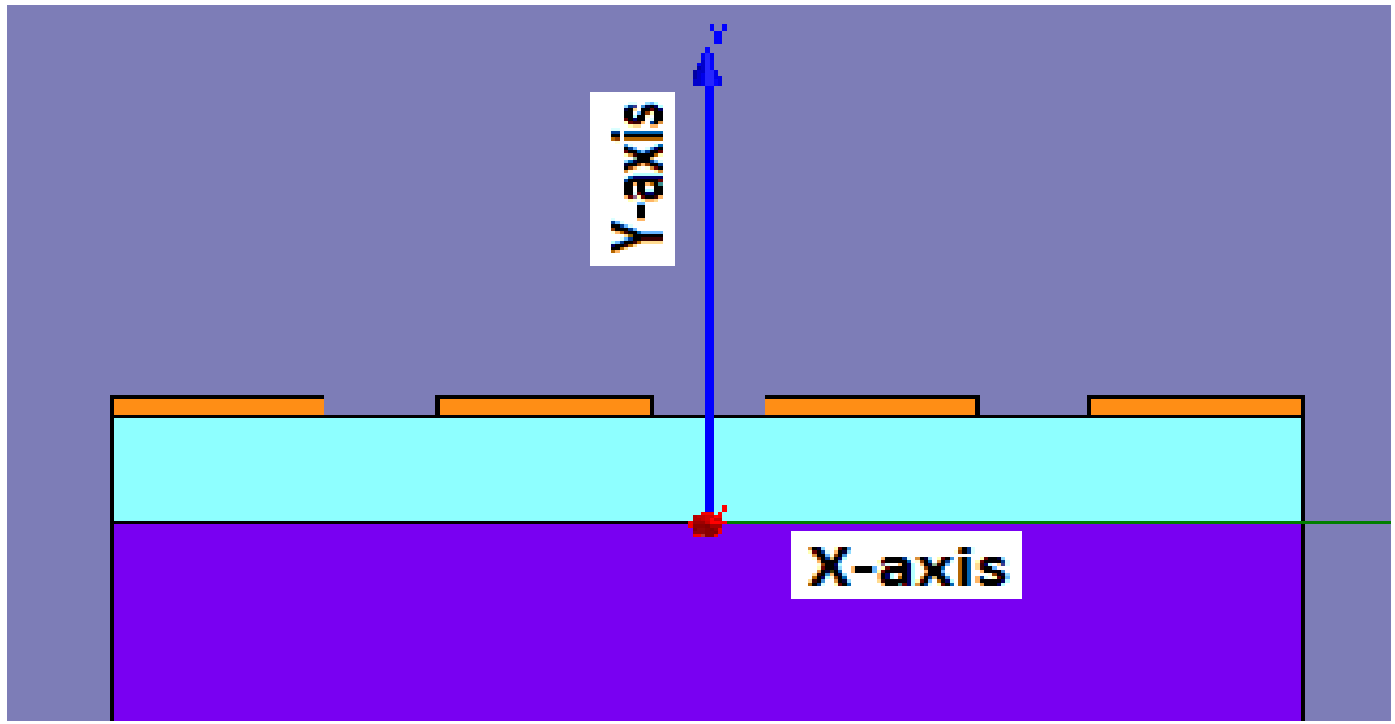

Figure 2.3: IDE cross section design

An AC conduction solver setup with alternating polarities of $25 \mathrm{mV}$ were assigned to the electrodes and the design was solved at $60 \mathrm{~Hz}$ with phase angle of $0^{0}$. In order to solve for the electromagnetic field of the model, the software solves the following equation:

$$
\nabla \cdot[\sigma \mathbf{E}+\mathbf{j} \omega \epsilon \nabla \phi(\mathbf{x}, \mathbf{y})]=\mathbf{0},
$$

where $f(x, y)$ is the magnitude and phase of the electric potential at each value of $\mathrm{x}$ and $\mathrm{y}, \omega$ is the angular frequency at which the potential is oscillating and $\sigma$ is the conductivity of the solution and $\varphi$ is the permitivity [20]. The above equation is derived from

$$
\nabla \cdot[\mathbf{J}+\mathbf{j} \omega \mathbf{D}]=\mathbf{0},
$$


$\mathrm{J}$ is the current density, D is the flux density, and $\mathrm{E}$ is the electric field strength [20]. The boundary is assumed to be insulated from any kind of outside electric/magnetic activity. The simulations show that most of the electric field is concentrated at a distance of few hundreds of nanometers above the surface which makes this geometry best fit for the sensor platform.Fig (2.4) shows the electric field current density distribution around th electrodes, which is just a few hundreds of nm above the surface of the electrodes. This is the region within which any small changes on the surface will result in a change in the corresponding electric signal. Different concentrations of whole cells or biomolecules bound to the surface yield different electrical impedance changes between the electrode structures and provide a means for a quantitive analysis of biomolecular interaction at the biosensor surface.

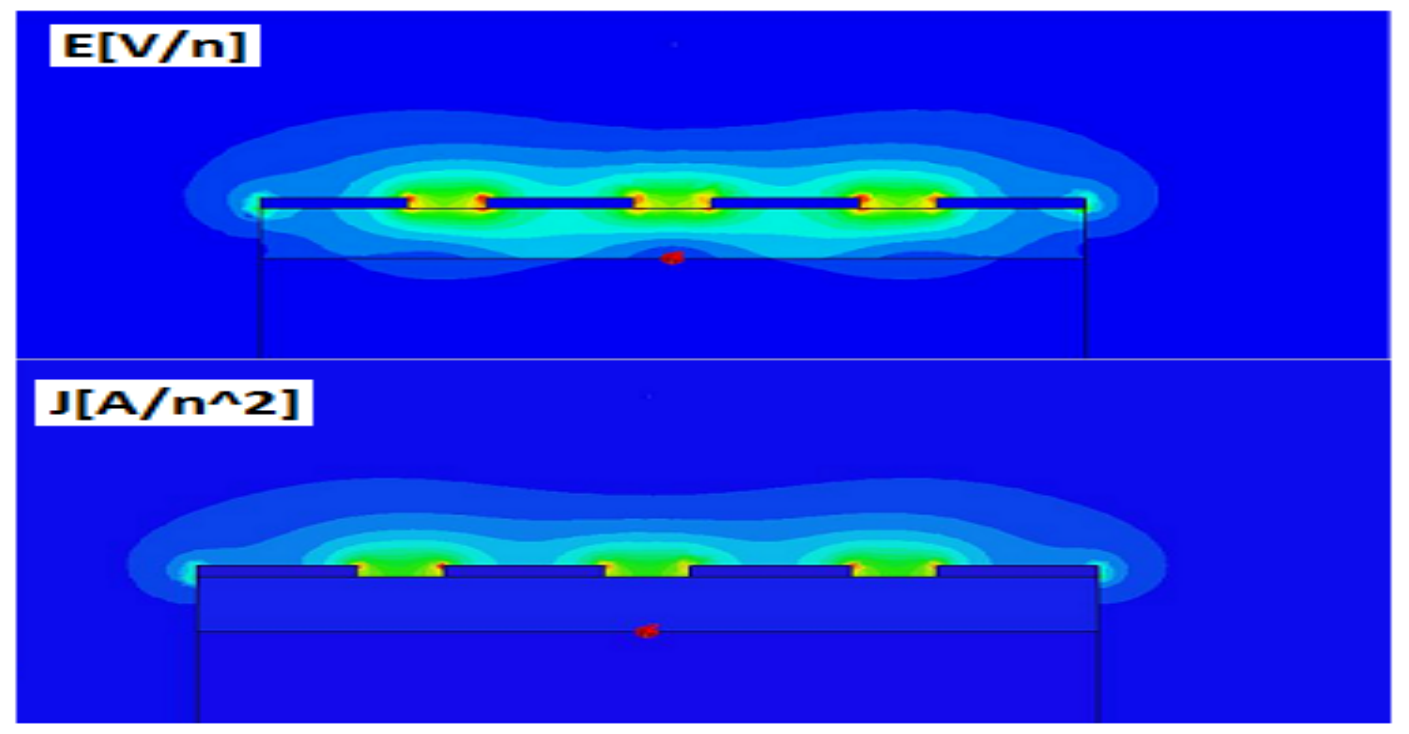

Figure 2.4: E-field strength(E) and current density $(\mathrm{J})$ distribution of the electrodes

Our lab has extensively studied the effect of IDE geometry on the distribution of electric field and current density for IDE's designed with different combinations of gap $(\mathrm{G})$,width (W) and height $(\mathrm{H})$ of the electrode digits. Device simulations showed 
that current density and electric field showed a large change in flux when gap between the electrodes was decreased below $800 \mathrm{~nm}$. The average electric field values at a distance of $50 \mathrm{~nm}$ from the electrode surface approximately increased by $41 \%, 112 \%$, $190 \%$, and $430 \%$ as the gap decreased to $500 \mathrm{~nm}, 300 \mathrm{~nm}, 200 \mathrm{~nm}$ and $100 \mathrm{~nm}$ respectively. But the average electric field value, determined at $800 \mathrm{~nm}$ gap for the IDEA fingers with W $1500 \mathrm{~nm}$ and H $200 \mathrm{~nm}$ as compared to a near $12.05 \%$ increase when gap was reduced from 1000 to $800 \mathrm{~nm}$ and have been published elsewhere [21,22] On the other hand, when gap was kept constant, the values of electric field and current density did not change drastically but registered a decrease in magnitude with an increase in the widths of the electrodes. Considering these values this work uses IDEA's with width of $1500 \mathrm{~nm}$, Gap $500 \mathrm{~nm}$ and height of $200 \mathrm{~nm}$ to achieve good sensitivity for IDEA biosensor platform.

\subsection{Electrochemical Impedance-Theory}

The electrodynamic theory of sinusoidally alternating currents and voltages is based on relatively simple laws. The relation between electrical current and applied voltage in the cases of ohmic resistors and capacitors following their phenomenological definition.

The alternating voltage is given by

$$
\mathbf{V}=\mathbf{V}_{\mathbf{m}} \sin \omega t
$$

then the resulting current through a resistance $\mathrm{R}$ follows Ohm's law

$$
\mathbf{i}=\left(\mathbf{V}_{\mathbf{m}} / \mathbf{R}\right) \operatorname{Sin} \omega \mathbf{t}
$$

The current passing a condenser with capacitance $\mathrm{C}$ is determined by the relation 
between potential 'V' and the charge ' $q$ ' on the plates:

$$
\mathbf{q}=\mathbf{C V}
$$

$$
\mathbf{i}=(\mathbf{d q} / \mathbf{d t})=\mathbf{C}(\mathbf{d V} / \mathbf{d t})=\omega \mathbf{C V}_{\mathbf{m}} \cos \omega \mathbf{t}=\omega \mathbf{C V m \operatorname { s i n }}(\omega \mathbf{t}+\pi / \mathbf{2}),
$$

Impedance is the ratio of a sinusoidal voltage, applied across two terminals of a measurement cell, to the sinusoidal component of the current flowing between the terminals that result from the applied potential difference [23]. Unless the system is purely resistive, impedance $\left(|Z|=\mathrm{V}_{\mathrm{m}} / \mathrm{i}_{\mathrm{m}}\right)$ is a complex quantity because the current will have a different phase from the applied voltage

$$
\mathbf{Z}=\mathbf{Z}^{\prime}+\mathbf{i} \mathbf{Z}^{\prime \prime}=|\mathbf{Z}| \mathbf{e}^{\mathrm{i} \theta}, \mathbf{i}=(-\mathbf{1})^{0.5}
$$

if we consider a resistance $\mathbf{R}_{\text {ser }}$ and capacitance $\mathbf{C}_{\text {ser }}$ [24] in series we find that the voltage is linked to the current through

$$
\mathbf{Z}=\mathbf{R}_{\text {ser }}-\mathbf{i} /\left(\omega \mathbf{C}_{\text {ser }}\right)
$$

Sometimes it is advantageous to analyze AC circuits in terms of admittance, $\mathbf{Y}\left(\mathrm{i}_{\mathrm{m}} /\right.$ $\mathrm{V}_{\mathrm{m}}$ ), which is the inverse of impedance, $1 / \mathrm{Z}$, usually represented by

$$
\mathbf{Y}=\mathbf{Y}^{\prime}+\mathbf{i} \mathbf{Y}^{\prime \prime}=|\mathbf{Y}| \mathbf{e}^{\mathrm{i} \theta}
$$

This concept is especially useful in the analysis of parallel circuits, because the overall impedance of parallel elements, e.g. $\mathbf{C}_{\text {ser }}, \mathbf{R}_{\text {ser }}$ is simply the sum of the 
individual admittances.

$$
\mathbf{Y}=\mathbf{1} / \mathbf{R}_{\text {ser }}-\mathbf{i}\left(\omega \mathbf{C}_{\text {ser }}\right),
$$

all combinations of elements (Z', Z"), (|Z|, $\theta),\left(\mathrm{C}_{\text {ser }}, \mathrm{R}_{\mathrm{ser}}\right),\left(\mathrm{Y}^{\prime}, \mathrm{Y}^{\prime}\right),(|Y|, \theta),\left(\mathrm{C}_{\mathrm{par}}\right.$, $R_{\text {par }}$ ) are equivalent and can be converted to one other [24].

\subsection{Electrochemical Impedance Spectroscopy}

Electrochemical Impedance spectroscopy (EIS) has long been employed for studying electrochemical systems. Impedance spectroscopy can predict aspects of the performance of chemical sensors and fuel cells, and it has been used extensively to investigate membrane behavior in living cells. It is useful as an empirical quality control procedure yet it can contribute to the interpretation of fundamental electrochemical and electronic processes. An analysis of the charge transport processes, likely to be present in an experimental cell, will often suggest an equivalent circuit of ideal resistors and capacitors and may account adequately for the observed EIS response.

A detailed physicochemical model of all the processes occurring on an electrodematerial systems may be unavailable or perhaps too complicated to warrant. In general one tries to show that the experimental impedance data $Z_{\mathrm{e}}(\omega)$ may be well approximated by the impedance $Z_{\mathrm{ec}}(\omega)$ of an equivalent circuit made up of ideal resistors, capacitors and various distributed circuit elements.

\subsubsection{Equivalent circuit Model}

Impedance methods are quite powerful, in that they are capable of characterizing physicochemical processes of widely differing time constants, sampling electron transfer 


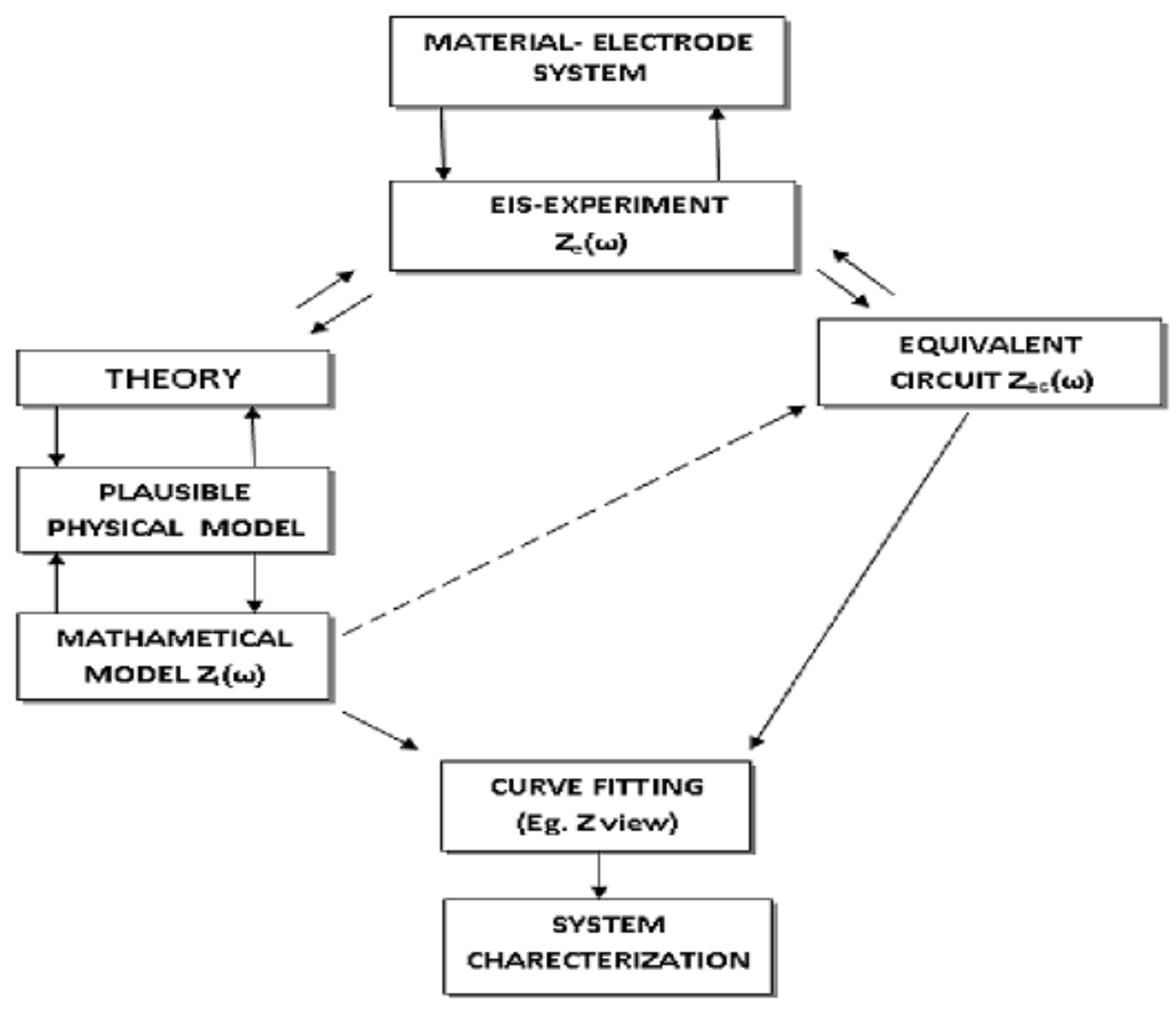

Figure 2.5: EIS Flow chart

at high frequency and mass transfer at low frequency. The impedance of an electrochemical cell is then the ratio of the small applied sinusoidal AC voltage excitation (typically $5-25 \mathrm{mV}$ ) to the measured current response is determined. The in-phase current response determines the real (resistive) component of the impedance, while the out-of-phase current response determines the imaginary (capacitive) component. This electrochemical cell performance can be represented by an equivalent circuit of resistors and capacitors that pass current with the same amplitude and phase angle that the real cell does under a given excitation. 


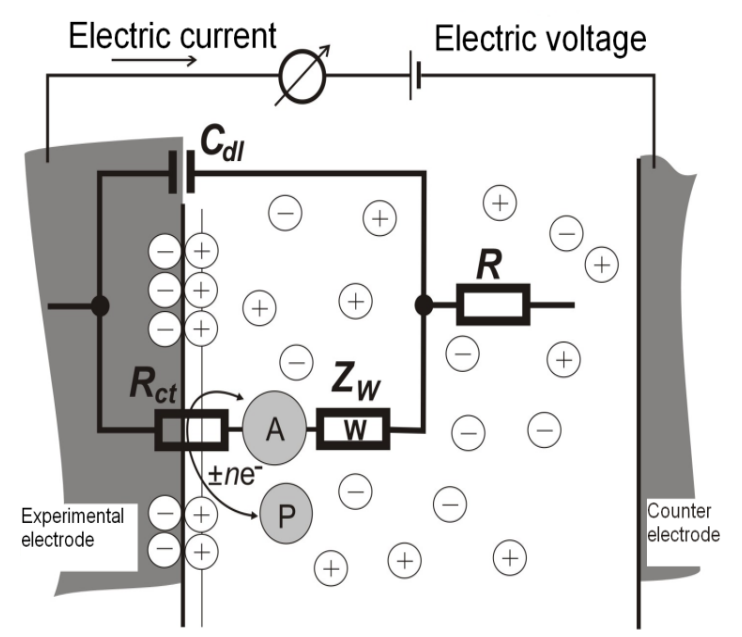

Figure 2.6: Randles Circuit of electrochemical cell(R-resistance of electrolyte, $\mathrm{C}_{\mathrm{dl}^{-}}$dou- $^{-}$ ble layer capacitance, $\mathrm{R}_{\mathrm{ct}^{-}}$charge transfer resistance, $\mathrm{Z}_{\mathrm{w}}$-warburges resistance) [10]

Above equivalent circuit models (a,b,c) represents a Randles equivalent circuit as applied to in an electrochemical cell. The resistance $R_{s}$ arises from the finite conductance of the ions in bulk solution, and thus is generally not affected by binding. The capacitance between the metal electrode and ions in solution, $\mathrm{C}_{\text {surf }}$, can be modeled as a series combination of the surface modification capacitance and the double layer capacitance. The component due to surface modification can be thought of as a parallel plate capacitor whose capacitance depends on the thickness and dielectric constant of the probe layer and is given by $\mathrm{C}=\epsilon_{r} \epsilon_{o} A / t$ where $\epsilon_{r}$ is the relative dielectric constant, $\epsilon_{r}$ is the dielectric constant of free space, A is the electrode area and $\mathrm{t}$ is the insulator thickness. There will be charge leakage across the double layer due to electrochemical reactions taking place at the interface which can be incorporated in terms of the charge transfer resistance, $R_{c t}$. The charge transfer resistance is a twofold process (1) the energy potential associated with the oxidation reaction event at the electrode along with (2) the energy barrier of the redox species reaching the 


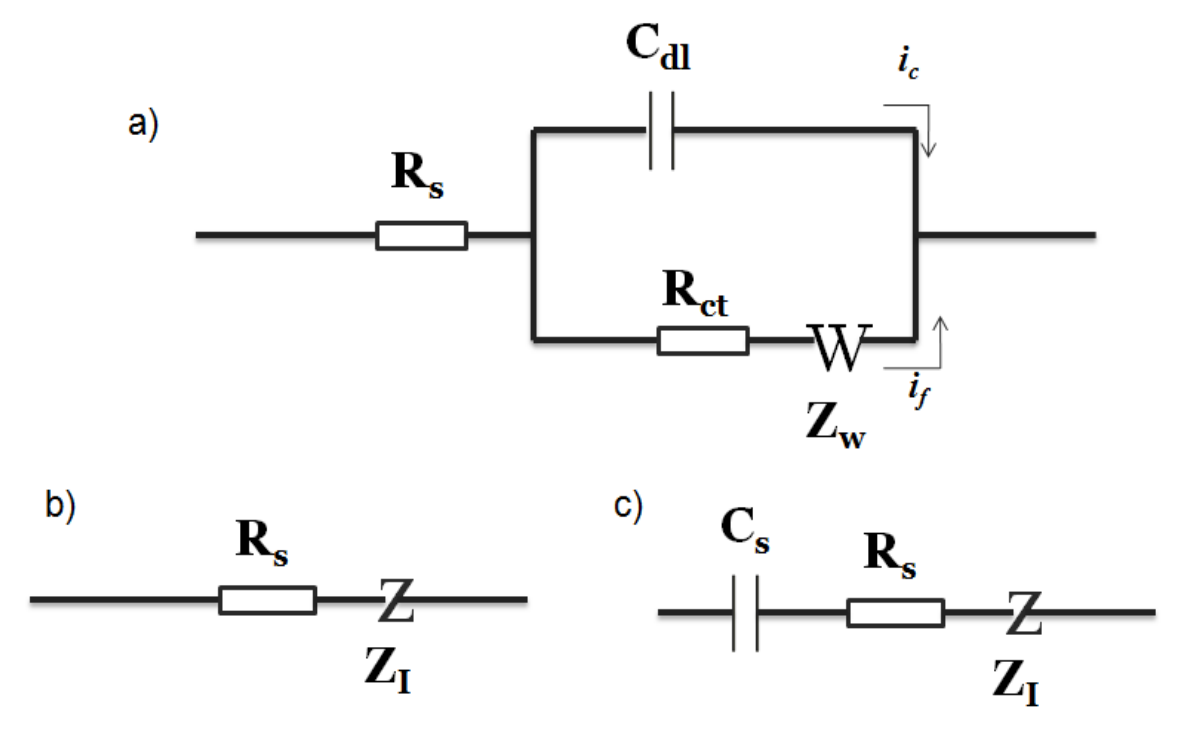

Figure 2.7: Equivalent Circuit Model [24]

electrode due to electrostatic repulsion or steric hindrance. Impedance due to diffusion of ions to the interface from the bulk of the electrolyte is often referred to as the Warburg impedance, $Z_{\mathrm{w}}$. It is only of physical significance in faradaic EIS and is only appreciable at low frequencies, is affected by convection and has a phase shift of $-45^{\circ}$. The parallel elements $\left(\mathrm{C}_{\mathrm{dl}}, \mathrm{R}_{\mathrm{ct}}, \mathrm{Z}_{\mathrm{w}}\right)$ of the interface impedance, $\mathrm{Z}_{\mathrm{I}}$, are introduced because the total current through the working interface is the sum of the distinct independent contributions from the faradaic processes, $i_{f}$, and double layer charging, $i_{c}$. All the currents must pass through the uncompensated solution resistance, and therefore $R_{\mathrm{s}}$ is inserted as a series element to represent this effect in equivalent circuit. In contrast to solution resistance and the double layer capacitance, which are nearly ideal circuit elements, the components of the faradaic impedance, $\mathrm{R}_{\mathrm{ct}}$ and $\mathrm{Z}_{\mathrm{w}}$ are not ideal, because they change with frequency and are dependent on the dielectric and insulating features at the electrode/electrolyte interface [25].In order to design a sensitive sensor with wide dynamic range, the insulating layer should be thin enough and/or have a 
high dielectric constant. otherwise, capacitance changes originating due to the binding of the analyte to the receptor might not dominate the total capacitance.In addition the insulating layer should be pin-hole free, stable with time, provide functional groups for immobilization of the receptor.Sometimes, particularly when the electrode surface is rough, the electronic properties of the interface cannot be described sufficiently well with a capacitive element, and a constant phase element $(\mathrm{CPE})$ should be introduced instead of $\mathrm{C}_{\mathrm{dl}}$, fig $(2.8)[17-18]$

$$
\mathrm{CPE}=\mathbf{A}^{-1}(\mathbf{j} \omega)^{-\mathbf{n}} .
$$

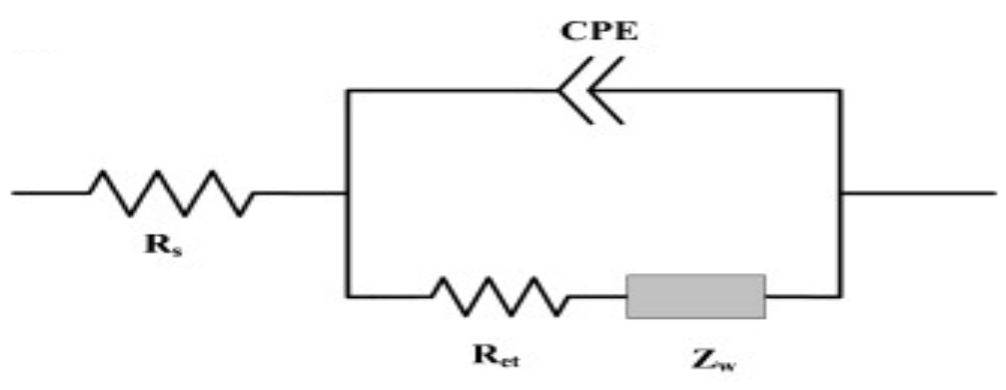

Figure 2.8: Equivalent Circuit Model with CPE

The constant phase element reflects non-homogeneity of the layer, and the extent of the deviation from the Randles and Ershler model is controlled by the parameter $\mathrm{n}$ in equation (2.11).Introduction of $\mathrm{CPE}$ element into an equivalent electronic circuit instead of simple capacitance was proved to be important for the modeling of primary protein layers on an electrode surface [18].Mathematically CPE counts as more than generalized electric element, consisting of group of parallel or branched resistivecapacitive transmission lines, for adjusting deviations originating in surface heterogeneities, the extent of the deviation being controlled by the parameter $\mathrm{n}(n \leq 1)$. 


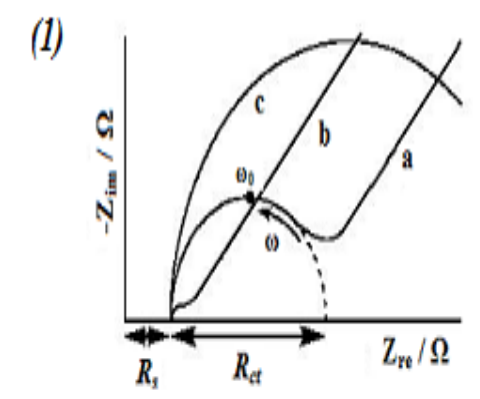

(2)

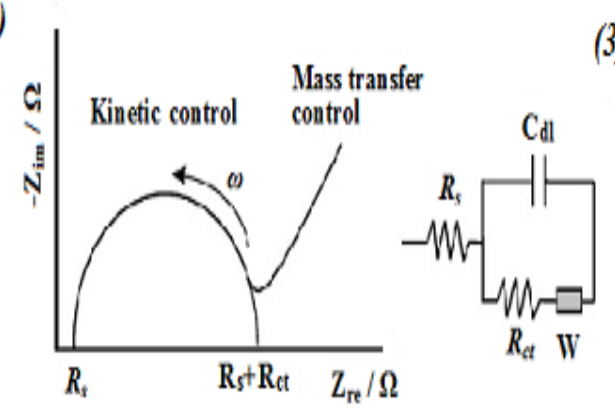

(3)

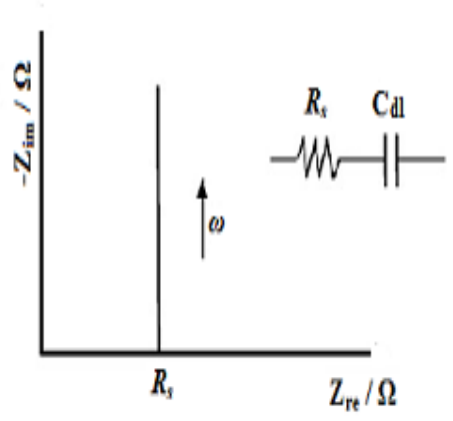

Figure 2.9: Nyquist plots and corresponding equivalent circuits [18,26]

In practice the presence of $\mathrm{CPE}$ instead of $\mathrm{C}$ means that the observed capacitance of the system is frequency dependent [18]. Therefore the two circuit elements most commonly used as indication of affinity binding are $\mathrm{C}_{\mathrm{dl}}$ for non faradaic biosensors and $\mathrm{R}_{\mathrm{ct}}$ for faradaic ones $[17,18,25-27]$. A typical shape of a faradaic impedance spectrum(Nyquist plot) includes a semicircle region lying on the axis followed by a straight line, curve a (fig2.9(1)). The semicircle portion, observed at higher frequencies, corresponds to the electron transfer-limited process, where as the linear part is characteristic of the lower frequency range and represents the diffusionally limited electrochemical process. The impedance spectrum would only include the linear part in case of fast electron transfer process curve b (fig(1)), where as a very slow electron-transfer step results in a large semicircle region that is not accompanied by a straight line, curve c 
(fig2.9(1)) [18]. The semicircle diameter equals to electron transfer resistance $\mathrm{R}_{\mathrm{ct}}$. The intercept of the semicircle with the $Z_{\text {re }}$ axiz at high frequencies $(\omega \rightarrow \infty)$ is equal to the solution resistance, $\mathrm{R}_{\mathrm{s}}$. Extrapolation of the circle to the lower frequencies yield an intercept corresponding to $\left(R_{\mathrm{s}}+R_{\mathrm{ct}}\right)$. One of the most important parameter governing the technique is the applied frequency. At very high frequencies $(f \geq 100 \mathrm{KHz})$ inductance of the electrochemical cell and connecting wires could contribute to the impedance spectra. At lower frequencies the impedance value is dominated by the DCconductivity of the electrolyte solution. Thus, the analytically meaningful impedance spectra are usually recorded at frequencies where they are mainly controlled by the interfacial properties of modified electrodes $(10 \mathrm{mHz} \leq \mathrm{f} \leq 100 \mathrm{KHz})$ [18]. 


\section{Chapter 3}

\section{Optimization of 3D IDEA's as Biosensor}

\subsection{Device Characterization:Sensitivity to Electrochemical Environment}

An experiment was designed to gauge the sensitivity of the IDEA to electrochemical environment. This was achieved by using three different concentrations of Potassium Ferri-Ferro cyanide redox pair $\left(\mathrm{K}\left[\mathrm{Fe}[\mathrm{CN}]_{6}\right]^{-3 /-4}\right)$. The solutions were made in deionized water(DI). The chips were thoroughly rinsed with DI water to make sure the surface were free of any debris. the chips were air dried and mounted on the chip mount and the Plexi glass channel was positioned to sit on top of the electrode structures, the clamps were tightened to make sure there were no leaks in the channel. The impedance analyser was setup and connected to the computer to take readings. The probe pins were carefully lowered to address the electrode structures. The concentrations of Potassium Ferri-Ferro cyanide redox pair solutions used for this experiment were $0.01 \mathrm{M}, 0.001 \mathrm{M}, 0.0001 \mathrm{M}$. The impedance analyzer was setup to collect data at $100 \mathrm{~Hz}$ to $100 \mathrm{KHz}$ with a DC bias of $0.215 \mathrm{mV}$ and an $\mathrm{AC}$ bias of $25 \mathrm{mV}$. The collected data was stored in the computer in the form of an Excel file. The data was further analyzed and the Nyquist plots were plotted as shown below.

The Nyquist plots showed that the diameter of the semicircle varied with concentration, which indicates that the electrodes were sensitive to the electrochemical 


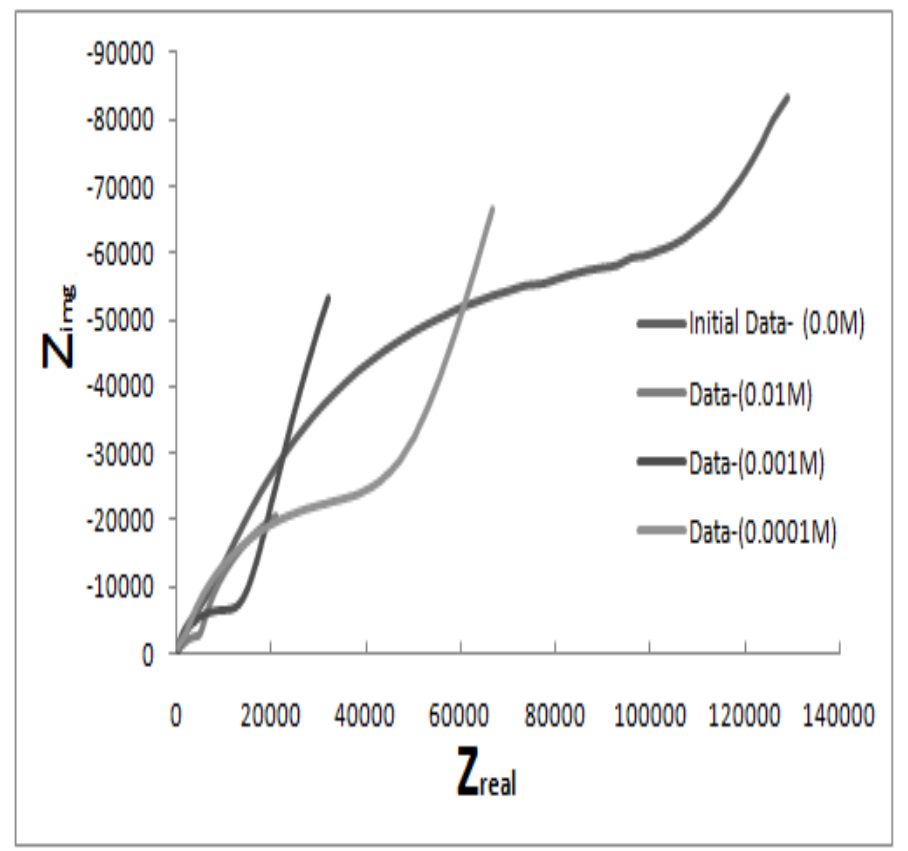

Figure 3.1: Niquist plots at different concentration of $\mathrm{K}\left[\mathrm{Fe}[\mathrm{CN}]_{6}\right]^{-3 /-4}$

concentration. In the above case the diameter decreases with increasing concentration because as the ionic concentration increased there were more number of ions traveling from the anode to the cathode and the current flow is increased therefore resistance decreases. The curve with the bigger diameter is the one with DI water which contains close to zero ions, therefore there is very little current flow i.e more resistant. In order to infer the resistance values from the plots we used a fitting softwares to fit the curves and get the approximate resistance $\left(R_{c t}\right)$. This was achieved by using $Z$-view software. It is a commercially available software to perform fitting analysis by assigning the circuit elements that could possibly be the best circuit to represent the curve. Snapshot of the Z-view software template is shown in Fig.3.2.

The data from the Excel sheets was processed and the real, imaginary impedance and the average $\mathrm{Z}$ and theta values were calculated and stored in a format acceptable to the Z-view software. Once the data was loaded, a circuit approximation was drawn 


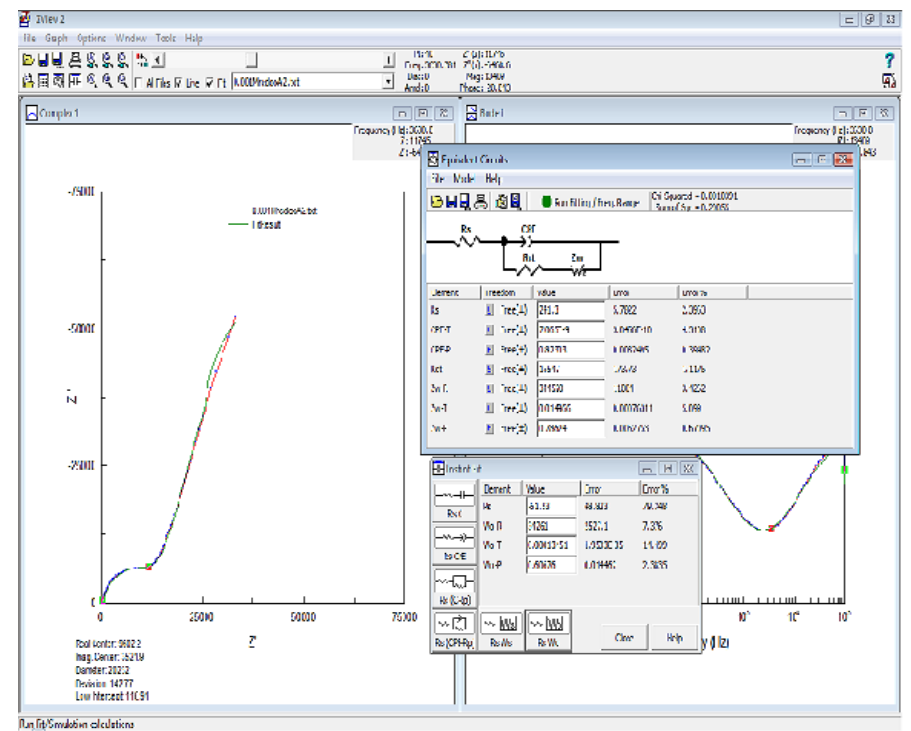

Figure 3.2: Z-view software template

to represent the graph and the circuit was then simulated. The software automatically generates the corresponding circuit element values. These circuit element values are then used to simulate the circuit and obtain the closest fit to the curve. Once the curve is perfectly fit the corresponding circuit element values can be used to make conclusions. The fig 3.3 shows the $R_{c t}$ values and the percentage errors which in this case is $5 \%$.

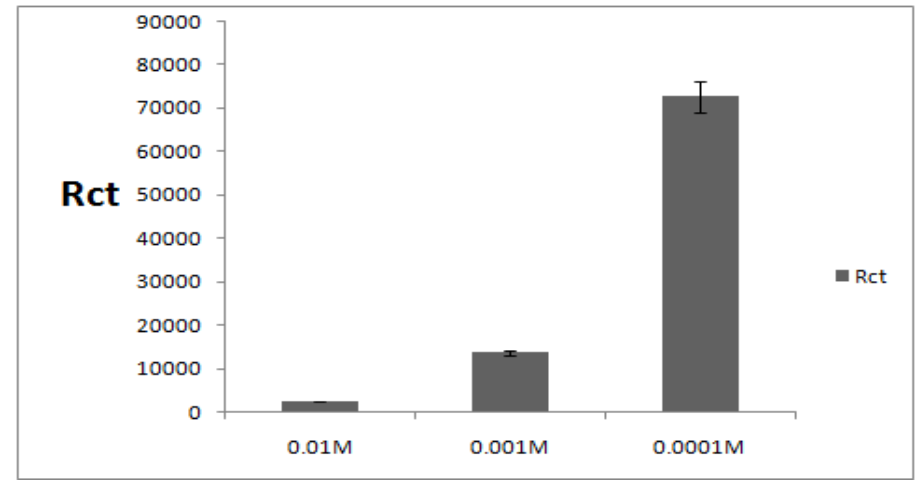

Figure 3.3: Plot showing $\mathrm{R}_{\mathrm{ct}}$ values obtained form Z-view software 


\subsection{Biofunctionalization of IDEA's}

\subsubsection{Surface Modification's}

Antigen and antibody immobilization is vital for successful development of a biosensor. In some experimental situations it is not feasible to immobilize antigen or antibody directly on to the metal electrode surface. To over come this situation and to mediate a reaction which is kinetically unfavorable at an unmodified electrode surface, surface modification is necessary. The present immobilization methods are mainly based on silanized layer, polymer membrane, Langmuir-Blodget film and self-assembled monolayer (SAM). Self-Assembled Monolayers (SAM) provides one of the simplest route to functionalize electrode surfaces by organic molecules containing free anchor groups such as thiols, disulphides, amines, silanes or acids. The monolayer produced by selfassembly are reproducible, ultra thin and well-oriented and are suitable for further modification with antibodies, which has potential in improving detection, sensitivity, speed and flexibility, which makes them particularly suitable for developing applications in areas of biosensors. Fig 3.5 in section 3.2.2 shows the structure of SAM on a electrode, which contains a head group and a functional group. The functional group can be further modified to attach to the specific reagent. The chain length of the SAM is about 3-5 nm which makes this platform more advantageous in biosensor applications $[28,29]$.

Self-assembled monolayers of alkanethiols on gold are one of the best available surfaces for accomplishing the functionalization and patterning necessary for fabricating a biosensor. There are various types of strategies available to couple the biomolecule to the electrode surface as follows:

- Covalent attachment of enzymes to the surface of the monolayer containing some 
free terminal groups like amines through amide linkage formations or attachment through some cross linker with the surface functional groups such as gtluteraldehyde.

- Non covalent coupling between the monolayers and the enzymes via electrostatic, hydrophobic and hydrophilic interactions.

- Through affinity interaction of receptor protein and recognition pairs of antigenantibody pairs. Biotinavidin is one unique example, leading to this type of a surface immobilisation of enzymes.

Advantages of the SAM's include:

- Easy formation of ordered, pinhole free stable monolayers.

- Flexibility to design the head group of the SAM's with various functional groups in ordered to achieve the desired surface.

- Only minimum amount of biomolecule is required for immobilization and reasonable stability for extended periods allows reliable measurements [28,29].

\subsubsection{Immobilization}

The chips used in this work were been fabricated by SHARP Laboratories at Camas, Washington. Initially experiments were conducted on the electrodes with different width and gap ratios to optimize the dimensions of the electrode structures. Each chip consisted of eight sets of IDEA's which was divided into two groups, each group with 4 IDEA arrays. Each electrode array structure had 39 digits from each side of width of $1500 \mathrm{~nm}$, gap of $500 \mathrm{~nm}$ and height of $200 \mathrm{~nm}$. These chips were plasma cleaned so there was no need for further cleaning with pirana solution, just a DI wash was 
sufficient. Then the chips were optically and electrically tested for diffective electrodes. Selection of the chips was made based on the prerequisite that at least six out of the eight structures were functioning. Notes were taken and the working electrodes were marked for future reference.

Once the selection is done, the chips were subjected to the following chemical procedures to engineer the gold electrode surface to serve as a sensor

- The first chemical step was to immobilize Self-Assembled Monolayers on top of the gold electrode to allow the attachment of protein. This was achieved by immersing the cleaned chips in 100 mM Cysteamine HCL (Sigma M6500) in 95\% ethanol solution overnight.

- The chips were thoroughly washed for 3-5 times using ethanol followed by DI wash.

- Then the chips were treated with $10 \mathrm{mM}$ triethylamine (Sigma T0886) in DI water for 1 hour at room temperature, followed by DI wash.

- Then the chips were placed in $2.5 \%$ glutraldehyde for 2 hours at room temperature in phosphate buffer solution. This step was performed to activate the functional groups of the surface layers for covalent coupling with the antigen/antibody. 


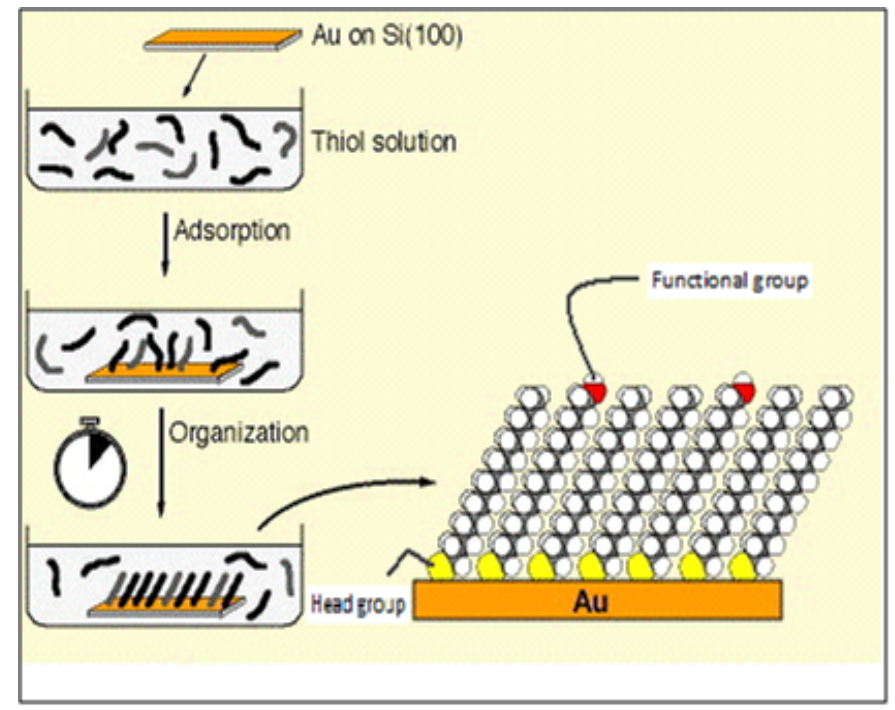

Figure 3.4: Formation of Self-Assembled Monolayers

\section{Protein Immobilization}

Immunoassays are commonly used to measure the concentration of a variety of hormones, allergens, viruses, and bacteria in clinical laboratory tests. Presently, much effort is put in the development of immunoassays, which allow monitoring of the antigen-antibody binding directly and which do not require the use of labeled species. The biomolecules have to be attached to a transducer surface with optimal preservation of their binding activity and specificity. Methods widely used are physical adsorption, covalent coupling, cross-linking, or entrapment in a gel network. This work focuses on the covalent coupling of the antigen and antibody. The protein immobiliztion is performed by immersing the electrodes in $5 \mu \mathrm{g} / \mathrm{ml}$ protein solution prepared in carbonate/bicarbonate buffer $(\mathrm{pH}=9)$ over night at room temperature, followed by wash with carbonate buffer. Then the chips were treated with $1 \%$ Ethanolamine (sigmaaldrich-411000) in carbonate buffer to neutralize the unreacted glutraldehyde groups, for 1 hour at room temperature. The chips were then treated with $1 \%$ PVP solution 


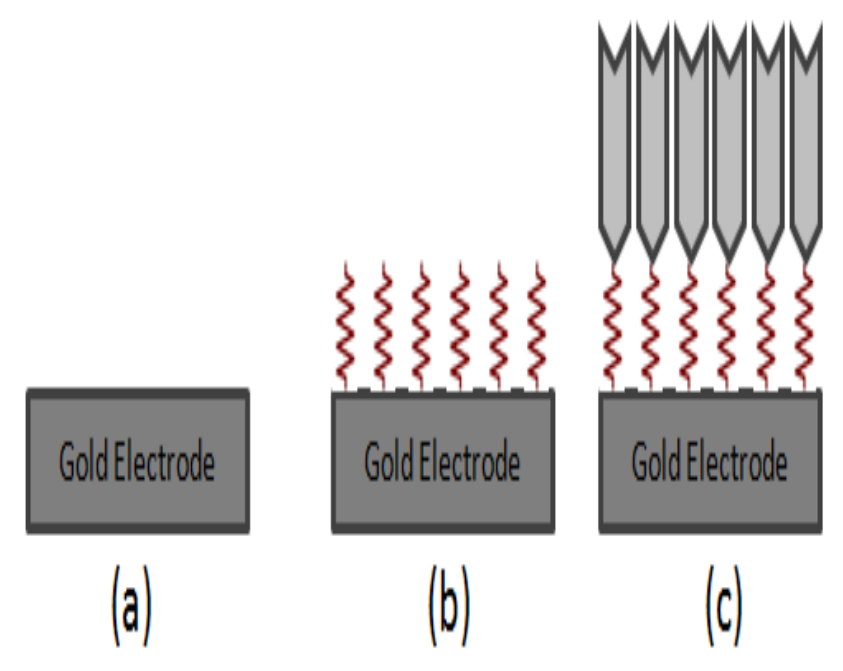

Figure 3.5: Protein Immobilization: (a) Bare Electrode (b) SAM layer (c) Protein immobilized on SAM

for 1 hour at room temperature to block the non specific binding sites. A large variety of antigen or antibodies can be linked to the SAM layer.

\subsection{IDEA's as Biosensor}

\subsubsection{Sensor Specificity}

In order to prove the affinity binding of the protein to the specific antibody in the test solution. An experiment was conducted to show that the antibody specifically binds to the protein available on the sensing layer, accounting to the impedance change, quantifying the amount of the antibody present in the solution which is a valuable information in immunoassay biosensors.Since the chip was designed with two groups of IDEAs(groups A and B as show in fig below), four of the electrodes were immobilized with Ovalalbumin (OVA) protein, mainly found in egg whites. The other four were immobilized with the protein of interest, Tissue Transgutaminaze (tTG). Anti-tTG 
antibody solution was prepared in the carbonate buffer in the concentration range of $0.1 \mu \mathrm{g} / \mathrm{ml}$ to $10 \mathrm{pg} / \mathrm{ml}$.

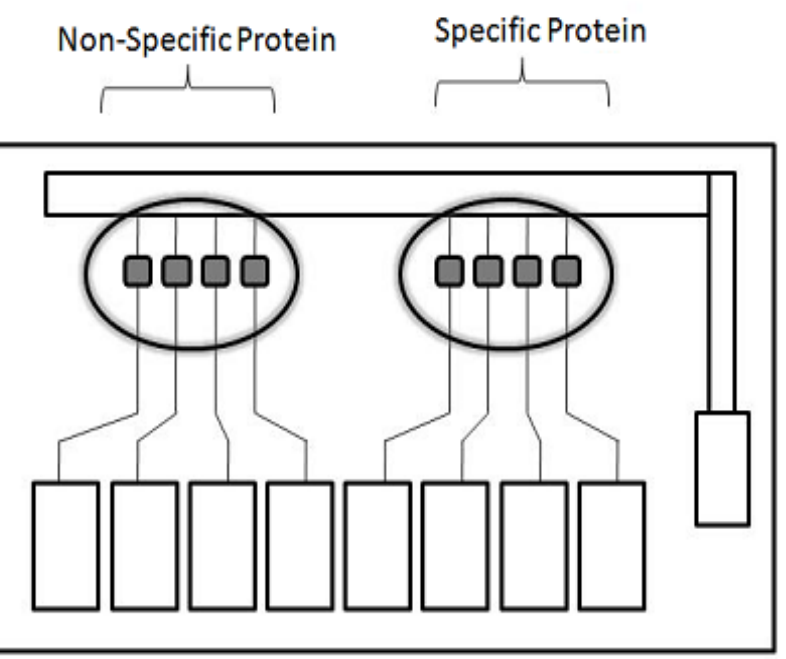

Figure 3.6: Chip showing two regions with different protein immobilization.

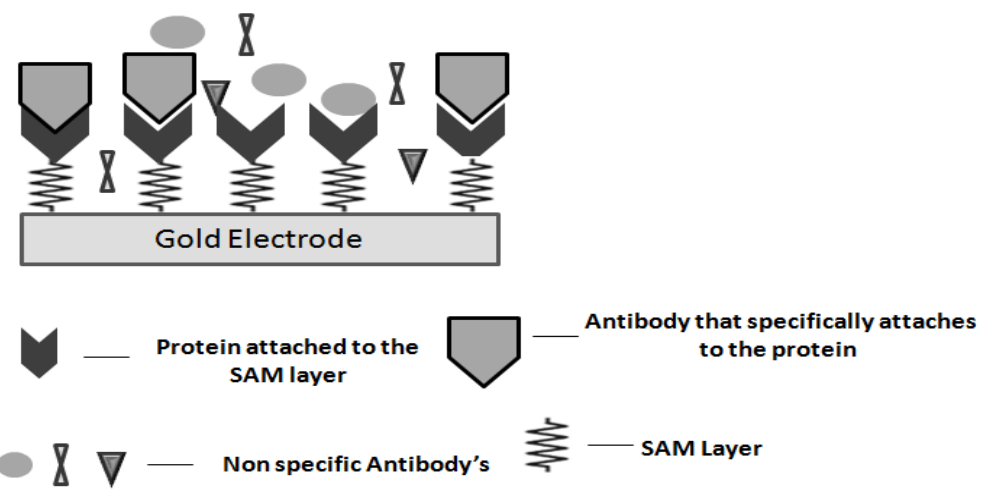

Figure 3.7: Specific vs Non-Specific binding. 
The experiment started with Electrochemical Impedance spectroscopy measurement of the protein immobilized electrodes in plain phosphate buffer solution(PBS) and the signal was termed $\mathbf{Z}_{\text {initial }}$ as a second step the antibody was incubated for about 10 mins followed by a PBS wash. The signal measured was termed $\mathbf{Z}_{\text {binding }}$. Then the sensor surface was regenerated using a low pH buffer called elution buffer and the signal reading was termed as $\mathbf{Z F i n a l . ~ T h e ~ s i g n a l ~ c h a n g e ~ b e t w e e n ~} \mathbf{Z}_{\text {initial }}$ and ZFinal was calculated by taking the average of the two values.

$$
\mathrm{ZAvg}=(\text { Zinitial }+ \text { ZFinal }) / 2
$$

the percentage difference between the initial and final values is then calculated by the following formula.

$$
\left(\left(\mathbf{Z}_{\text {Binding }}-\mathrm{ZAvg}\right) / \mathrm{ZAvg}\right) * \mathbf{1 0 0}
$$

It was observed that most changes in EIS readings took place below $1 \mathrm{KHz}$, so the histogram plots were plotted with the $\mathrm{dZ} \%$ change values at $500 \mathrm{~Hz}$. The plots clearly revealed that there was a clear difference in the specific and non specific binding patterns. The plots show that there is a huge signal difference at $10000 \mathrm{ng} / \mathrm{ml}$ and as the concentration is reduced the data follows the similar pattern. Clearly specific binding gives us a large signal than the non specific protein. At this point it is safe to say that this immobilization process provides good specificity. However there are some interchip irregularities, which will be addressed later. With this experiment we can shed light on two important things, one being the specificity and the other is sensitivity. This experiment also shows the lower detection limit or in other words sensitivity of this sensor platform. It will be safe to assume that the lower detection limit was $0.1 \mathrm{ng} / \mathrm{ml}$ which is a real good lower limit for immunoassays. 


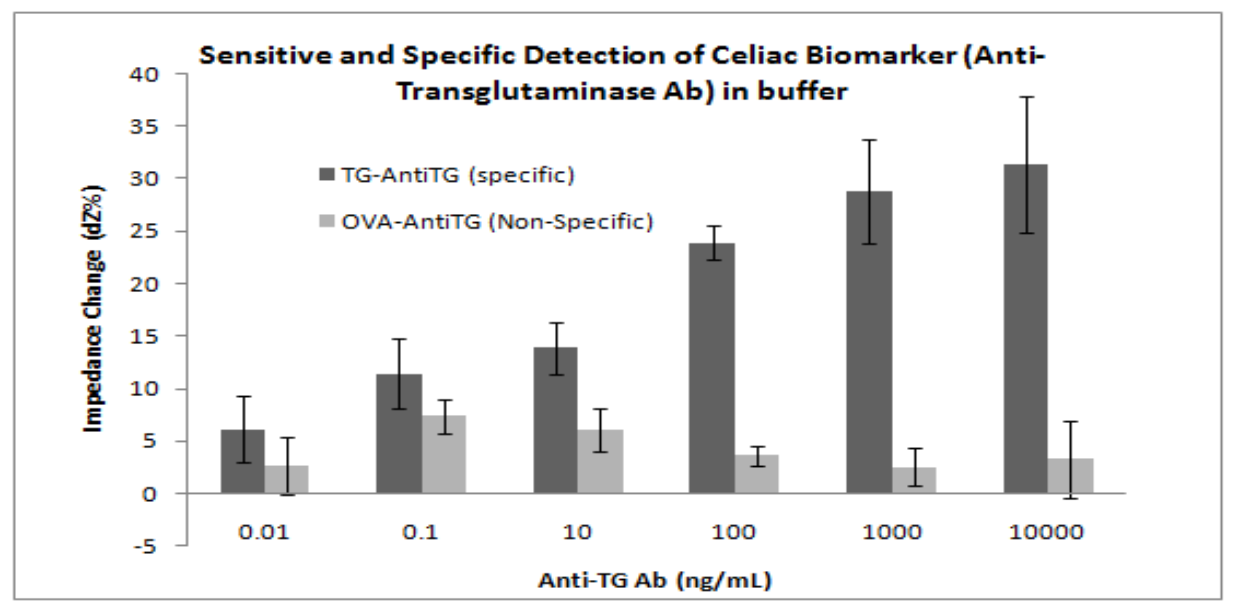

Figure 3.8: Plots showing Specific vs Non-Specific binding.

\subsubsection{Biosensor Vs Bioassay}

The difference between bioassays and the biosensors is pertained to the read out system. While bioassay is the generic term for a wide variety of assays that combine biological recognition element with a range of biochemical,physicochemical changes that can be read separately. The term biosensor is used exclusively for those systems that integrate the biorecognition elements with a physicochemical transducer system such as an electrochemical transducing system, and there by suitable for online measurements. The bioassays are slow since they require multiple steps in a lab environment however simple biosensors are promising alternative to traditional bioassays with the rapid online measurements, however have relatively low sensitivity compared to bioassays eg. ELISA. The application of nanotechnology has led to increasingly complex designs of biosensors, thereby creating some overlap with bioassays in terms of sensitivity. The biosensor platform discussed here has been engineered to achieve high sensitivity close, if not equal to that of a traditional ELISA [30]. An experiment was performed, which is discussed in the next section, to demonstrate this. 


\section{Bioassay: Enzyme Linked Immunosorption Assay(ELISA)}

To confirm the binding of antigen-antibody on the sensor surface a traditional ELISA bioassay was performed on the chips, the results were compared with those of the biosensor assay. For on-chip ELISA the chips were functionalized as mentioned before, tTG acting as the biological agent was immobilized on the sensing surface. These chips were then incubated with different concentrations of goat anti-TG antiserum in the flow channel for 10 mins followed by the PBST wash. An HRP-enzyme conjugated secondary antibodywas used to probe the capture of tTG specific antibodies on the chip surface, Rabbit anti-Goat-IgG HRP conjugated (Sigma, A5420) secondary antibody was subsequently injected into the channel at dilutions suggested by the manufacturer. After 20 mins the chips were subjected to a PBST wash and the flow channel was removed. As shown in the figure each chip was transferred to a petridish quadrant containing $5 \mathrm{~mL}$ of TMB/ $\mathrm{H}_{2} \mathrm{O}_{2}$ substrate system(Sigma, T0440).

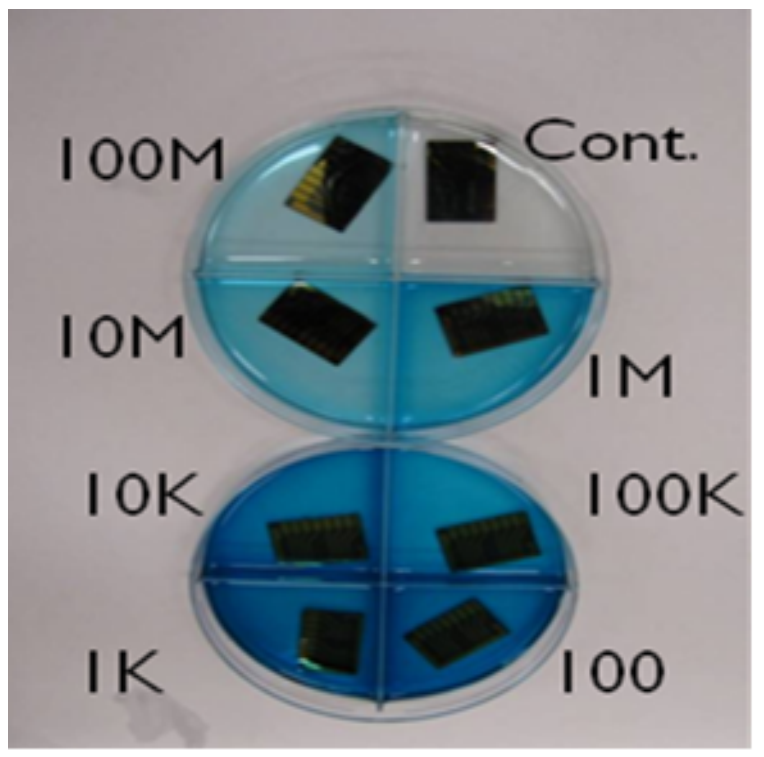

Figure 3.9: On-chip ELISA, the developed colour increase with decreasing dilution of serum samples indicating the presence of more Abs bound to the electrodes. 
The HRP chromogenic substrate reaction(appearence of blue color) was allowed to proceed for $20 \mathrm{~min}$, later $1 \mathrm{ml}$ of the blue solution was taken out of the petridish and the reaction was ceased by adding $0.5 \mathrm{ml}$ acidic stop solution $\left(1 \mathrm{~N} \mathrm{H}_{2} \mathrm{SO}_{4}\right)$. The conformation was obtained by the change of color from blue to yellow. The optical density(O.D) of yellow color solution was read at $450 \mathrm{~nm}$ wavelength using a spectrophotometer (Shima dzu UV-1800). The color intensity was proportional to the anti-TG antiserum dilution used.. The results are plotted in fig3.10. The signal (O.D.450 nm) changed from 0.307 to 2.7 over the serum dilution ranging from $10^{-7}$ to $10^{-2}$. Showing an increase of 8.8 times for a concentration change of five orders, with highest O.D. at lowest serum dilution that indicates more specific $\mathrm{Ab}$ is available to bind the antigen at lower dilutions [20].

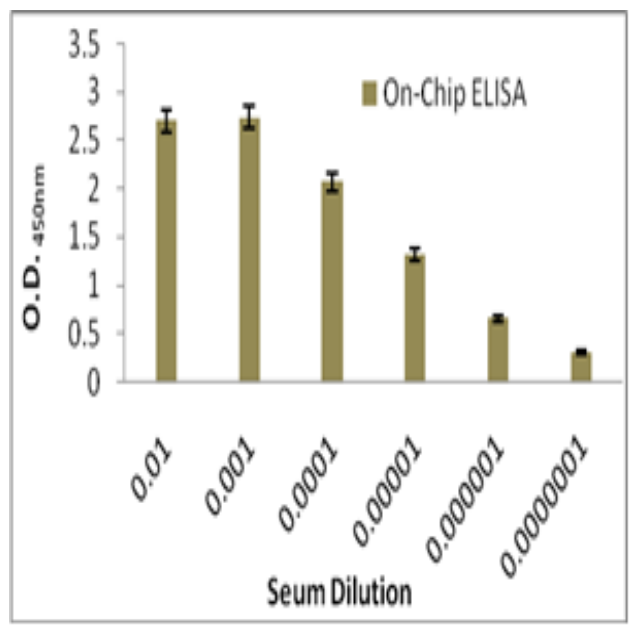

Figure 3.10: On-chip ELISA, the optical density at $450 \mathrm{~nm}$ was recorded and plotted against the serum dilutions. Please see text for details. 


\subsection{Biosensor Assay}

An experiment was performed to prove that the biosensor platform of interest achieved the sensitivity levels close to that of bioassay.The chips were functionalized with tTG antigen described earlier using SAM method. Different concentrations of anti-tTGserum were used to bind to the antibody on the sensor surface and then the EIS readings were taken according to the topology presented below and the data was stored in an Excel sheet and a Nyquist plot concentration profile was extracted as shown in the figure below.

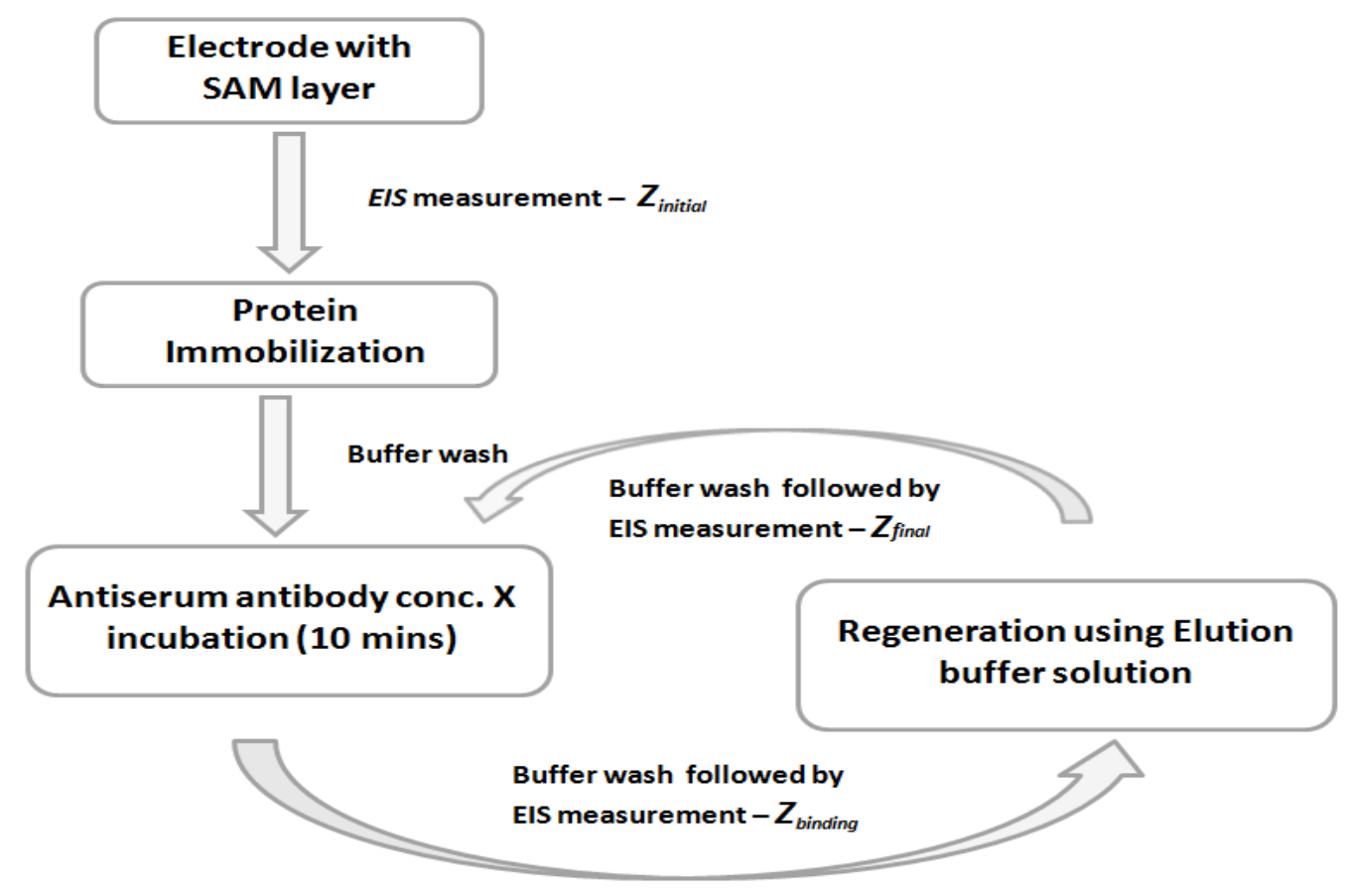

Figure 3.11: Topology for the experimental procedure.

It is clear from the plot that there is a noticeable change in the semicircular part of the Nyquist plot as the concentration changes, accounting for the $\mathrm{R}_{\mathrm{ct}}$ change that reflects the changes occurring at the sensor surface. The data obtained was analysed using Z-view software to extract the values of $\mathrm{R}_{\mathrm{ct}}$ that are plotted in fig3.13 against the 


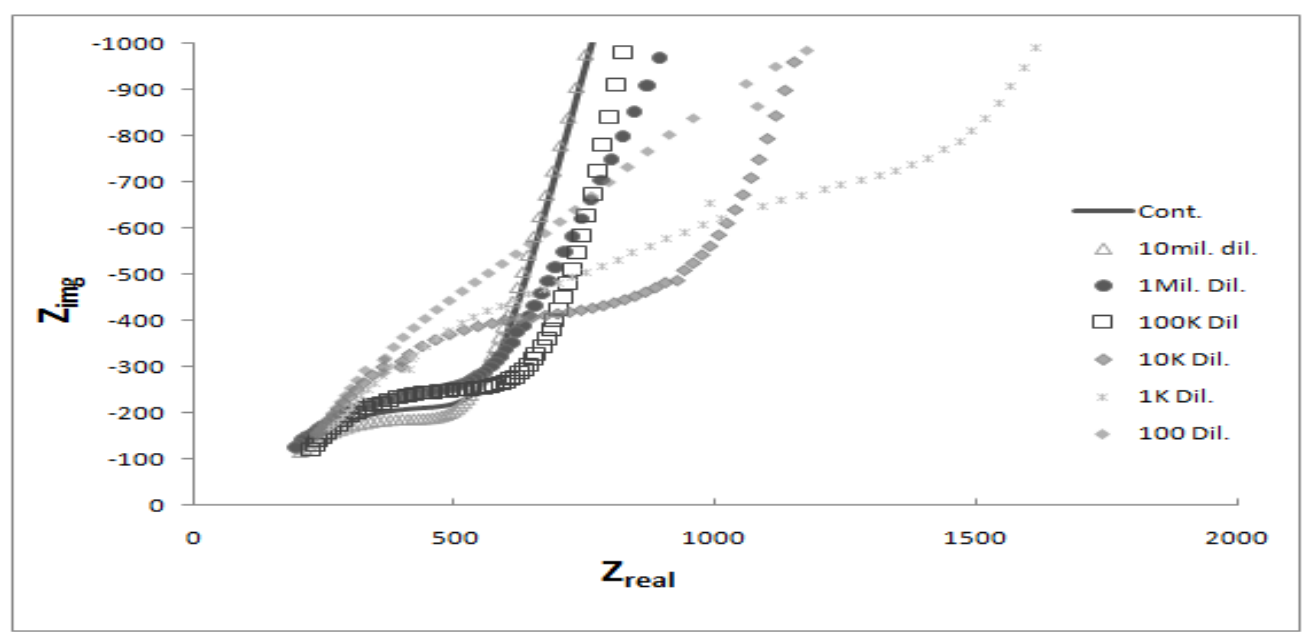

Figure 3.12: Nyquist Plots showing the concentration profile of Anti TG serum antibody.

serum dilution. A maximum average electron transfer resistance $\left(R_{c t}\right)$ of $1855 \Omega$ was determined for the least diluted serum sample and $494.7 \Omega$ at the maximum dilution. The change in signal was proportional to the change in available anti-tTG antibodies in the applied sample and the observed binding signal increased by 3.75 times when the concentration changed by five orders of magnitude i.e., from least dilution $\left(10^{-2}\right)$ to maximum dilution $\left(10^{-7}\right)$ of serum in PBST. Clearly ELISA with a signal change of 8.8 times is more sensitive than the IDEA biosensor for detection of Abs captured from the same samples concentration range 


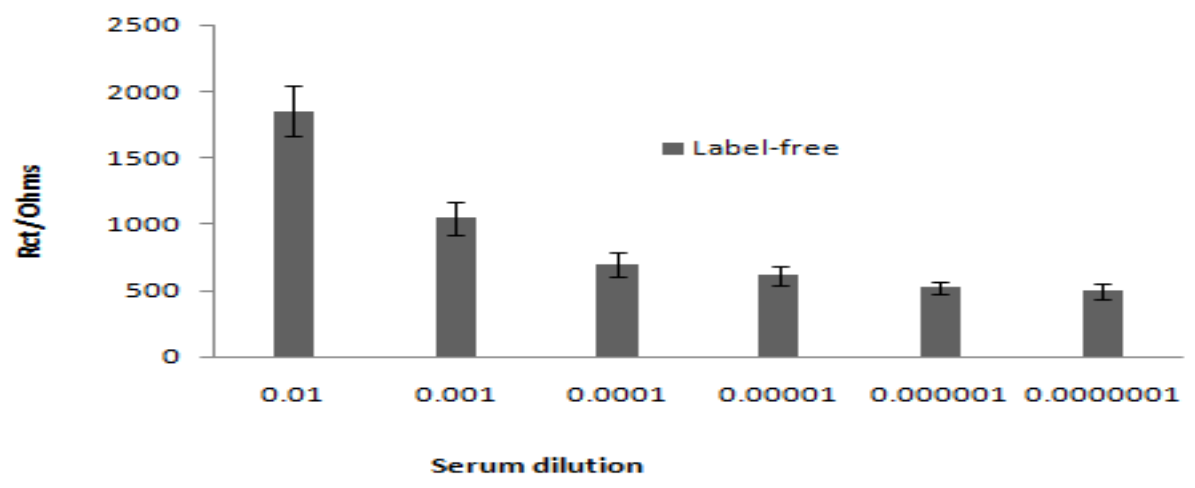

Figure 3.13: Plot showing $\mathrm{R}_{\mathrm{ct}}$ values corresponding to the relative impedance change with antiserum dilutions.

\subsubsection{Signal Enhancement using Nanoparticle conjugates}

Clearly a comparison of ELISA and the label free biosensor shows that the latter is less sensitive as compared to the traditional bioassay. In order to bring the sensor sensitivity close to that of the traditional ELISA assay, the use of labels for biosensor signal enhancement was considered. The unique properties of gold nanoparticles have stimulated the increasing interest for their application in interfacial biological recognition events with signal transduction and in designing biosensor devices exhibiting novel functions. The excellent biocompatibility, conductivity, catalytic properties and high density of gold nano particles facilitate extensive application of nano particles in the construction of electrochemical and piezoelectric biosensors with enhanced analytical performance with respect to other biosensors.Protein-A (Pr-A) conjugated Gold Nanoparticles are extensively used in immunoassays because Pr-A binds with a very high specificity with antibodies. Pr-A gold nanoparticle (PrA-GNP) conjugate was prepared as described below for final application in the NP-labeled biosensor assay. 


\section{Preparation of PrA-GNP labels}

Colloidal gold nano particles were prepared in the lab by heating $200 \mathrm{ml} 0.01 \%$ gold chloride solution up to the boiling point in a conical flask using a heating pad. $4 \mathrm{ml}$ of $1 \%$ Trisodium citrate was quickly added to the boiling solution. The color of the colloidal gold solution changed from blackish purple through dark purple to typical wine red. This colloidal gold solution was used to prepare the protein conjugated nanoparticles. The reason behind conjugating the nano particles with the protein is to increase the surface area for specific protein interaction which in turn increases the output signal from the transducer.

\section{Preparation of Protein Colloidal Gold conjugate.}

The conjugation of the Pr-A and the colloidal gold is done in three different steps.

- Determination of optimum $\mathrm{pH}$. The $\mathrm{pH}$ of the colloidal gold solution was adjusted in the range of 5.0 to 9.0 using $0.1 \mathrm{~N} \mathrm{HCl}$ or $0.2 \mathrm{~N} \mathrm{K2CO}$. Fixed amount of protein $\mathrm{A}(10 \mu \mathrm{l}$ of $1 \mathrm{mg} / \mathrm{mL})$ was taken in microtiter plate wells and $100 \mu \mathrm{L}$ of colloidal gold solution at different $\mathrm{pH}$ was added. After incubation for $15 \min$. $20 \mu \mathrm{L}$ of $10 \% \mathrm{NaCl}$ solution was added to each well. The color of the colloidal gold changes to bluish-black of flocculated gold solution where it was not stabilized by the protein.

- Determination of optimum amount. Colloidal gold solution was adjusted at optimum $\mathrm{pH}$ by taking 2-20uL(Different amount of protein was taken to determine the optimum amount of protein required per ml of colloidal gold solution for stabilization at optimum $\mathrm{pH}$ ) of protein solution and $100 \mu \mathrm{L}$ of colloidal gold solution (optimum $\mathrm{pH}$ adjusted) was added. After incubation for 15 min. stabilization of colloidal gold was checked by addition of $20 \mu \mathrm{L}$ of $10 \% \mathrm{NaCl}$ solution. 
The minimum amount of protein where no color change was observed was noted and protein amount required to stabilize the one $\mathrm{mL}$ of gold solution was determined by extrapolation.

- Conjugation of Protein Colloidal-gold Nanoparticles. The colloidal gold protein reagents were prepared by mixing the optimized amount of Protein-A with Colloidal gold nanoparticles adjusted to optimum $\mathrm{pH}$.

The experiment in the previous section was then performed using protein-conjugated nano particles to enhance the sensitivity by providing more surface area for the antiserum antibody to bind to the target molecules which is reflected by the change in the charge transfer resistance values.

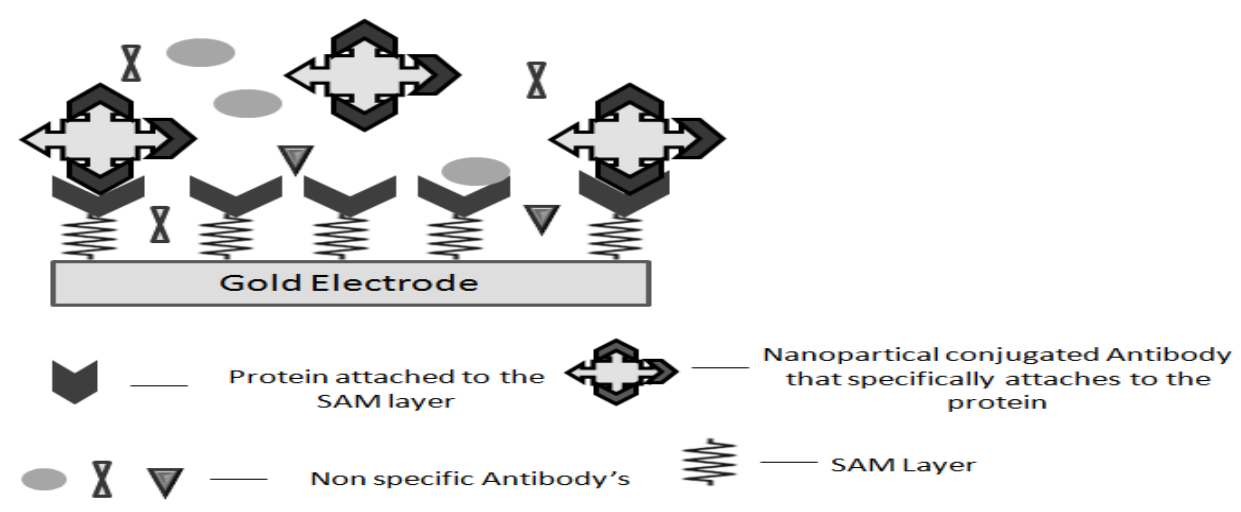

Figure 3.14: Protein conjugated nanoparticle binding to the electrode surface.

The experimental procedure was same as one in the previous section,with an additional step after the primary binding the signal enhancing PrA-GNP's were allowed to bind to the tTG specific Ab captured on the electrode surface. Data was collected in an Excel sheet and Nyquist plots were extracted from the data as shown below.

The graphs shown below consists of Niquistplots with and with out nanopartiles, One can clearly notice the shift in the diameter of the semicircle before and after binding 


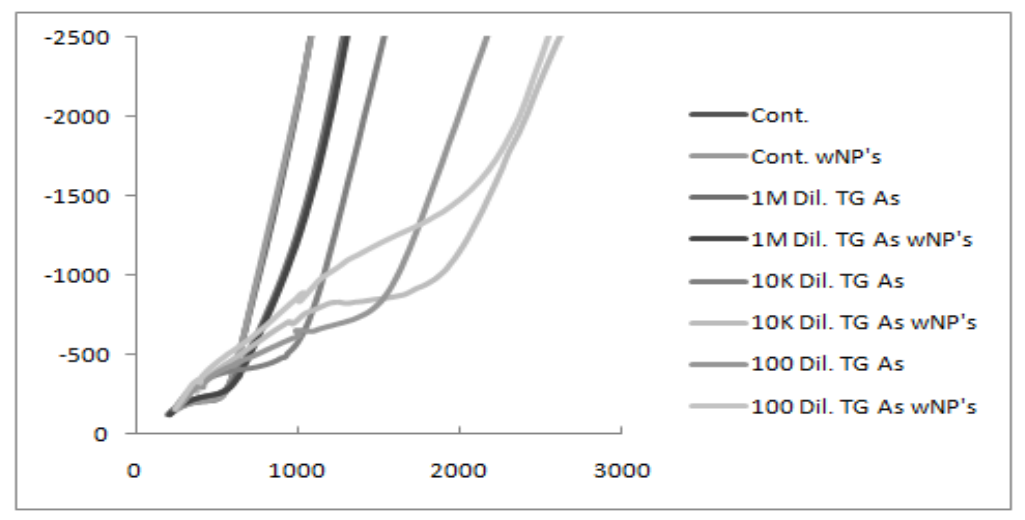

Figure 3.15: Nyquist plot with and with out nano particles.

of PrA-GNP at the same serum dilution, where the nano particle curves diameter is much larger than the one of the protein without nano particle. This proves that the increased sensitivity is achieved by the use of nano particles. The EIS data obtained was then processed with the Z-view software to get charge transfer resistance. The plot below shows a comparison of both protein only and protein with nano particle $R_{c t}$ values. The best fits for the curves were obtained using Z-view and The Rct values obtained from the Z-view analysis clearly show the signal enhancement achieved with the use of nano particles where the enhancement is higher at lower serum dilutions because more $\mathrm{Ab}$ is captured on the electrode surface with the PrA-GNPs, hence have more binding sites are available. The enhancement results in terms of $R_{c t}$ change with and without nanoparticle enhancement are plotted in the fig 3.17 below.

\subsubsection{ELISA vs Biosensor}

The histogram in fig3.18 shows the comparison of signal obtained with the label free PrA-GNP labeled biosensor assay and the traditional ELISA detection method.

These results support the statement made in the previous section, that the IDEA transducer can act as a biosensor platform capable of detection in the same sample 


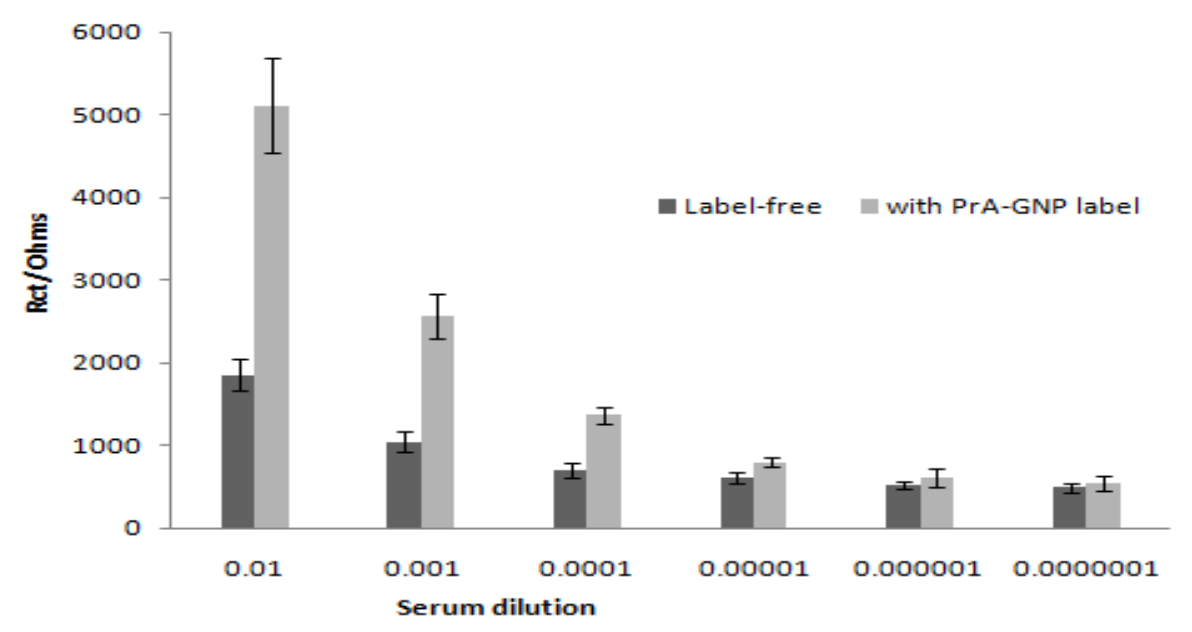

Figure 3.16: Plot showing the comparison of the $\mathrm{R}_{\mathrm{ct}}$ values with and with out nanoparticles.

concentration range however, labeling may be required to achieve the sensitivity closer to the traditional ELISA. . An indicator of the assay sensitivity is the slope of the assay curve. To compare the relative sensitivity of the ELISA with biosensor the signals were normalized with respect to the maximum signal for each of the three detection methods (fig. last). The slope was calculated using the best linear fit of the assay giving the slope of the curve shown as best fit to a linear equation i.e., $y=m x+c$, where $\mathrm{y}$ is signal, $\mathrm{x}$ is concentration and $\mathrm{m}$ is the slope with $\mathrm{c}$ as constant. The ELISA assay with a slope of 9.49 is the most sensitive and 3.6 times more sensitive than the label-free biosensor assay where the slope is just 2.63, this slope value increases to 9.15 with use of PrA-GNP labels for signal enhancement, thus bringing the biosensor assay sensitivity at par with ELISA. The biosensor platform holds several advantages over ELISA. It is possible to incorporate the whole system into a small hand held device that can be used as a point-of-care device eliminating the time taken by samples to be moved to central lab facilities and thereby improving healthcare management. Other advantages include ease of use, fast assay time and ability to incorporate changes to accommodate 


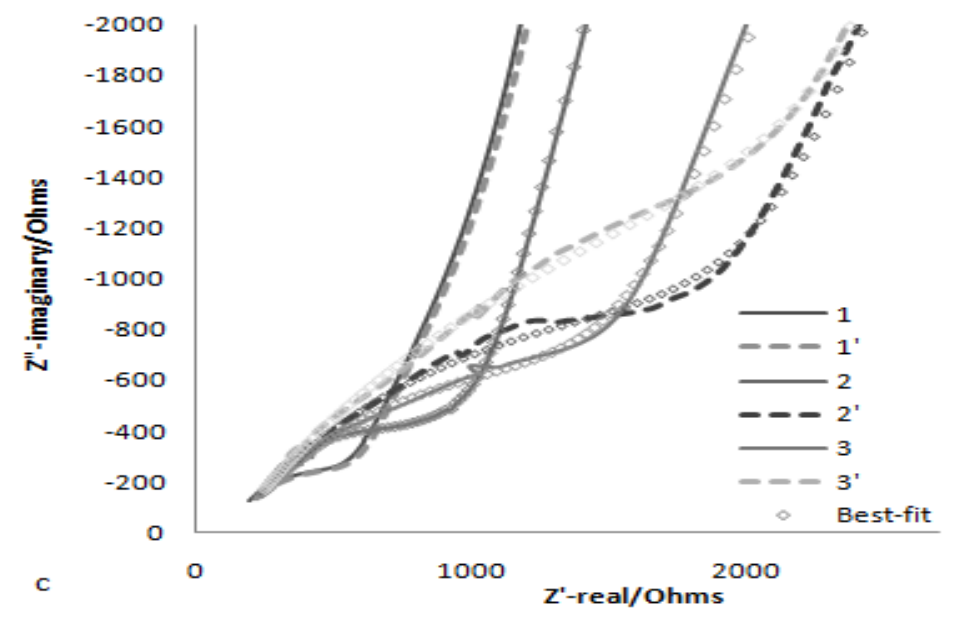

Figure 3.17: Fitting curves of Pr-NP data, here 1, 2, 3 are 1 Million, 10 K, 100 dilutions of the protein conjugated with NPs and 1', 2', 3' are the fit curves obtained from Z-view analysis.

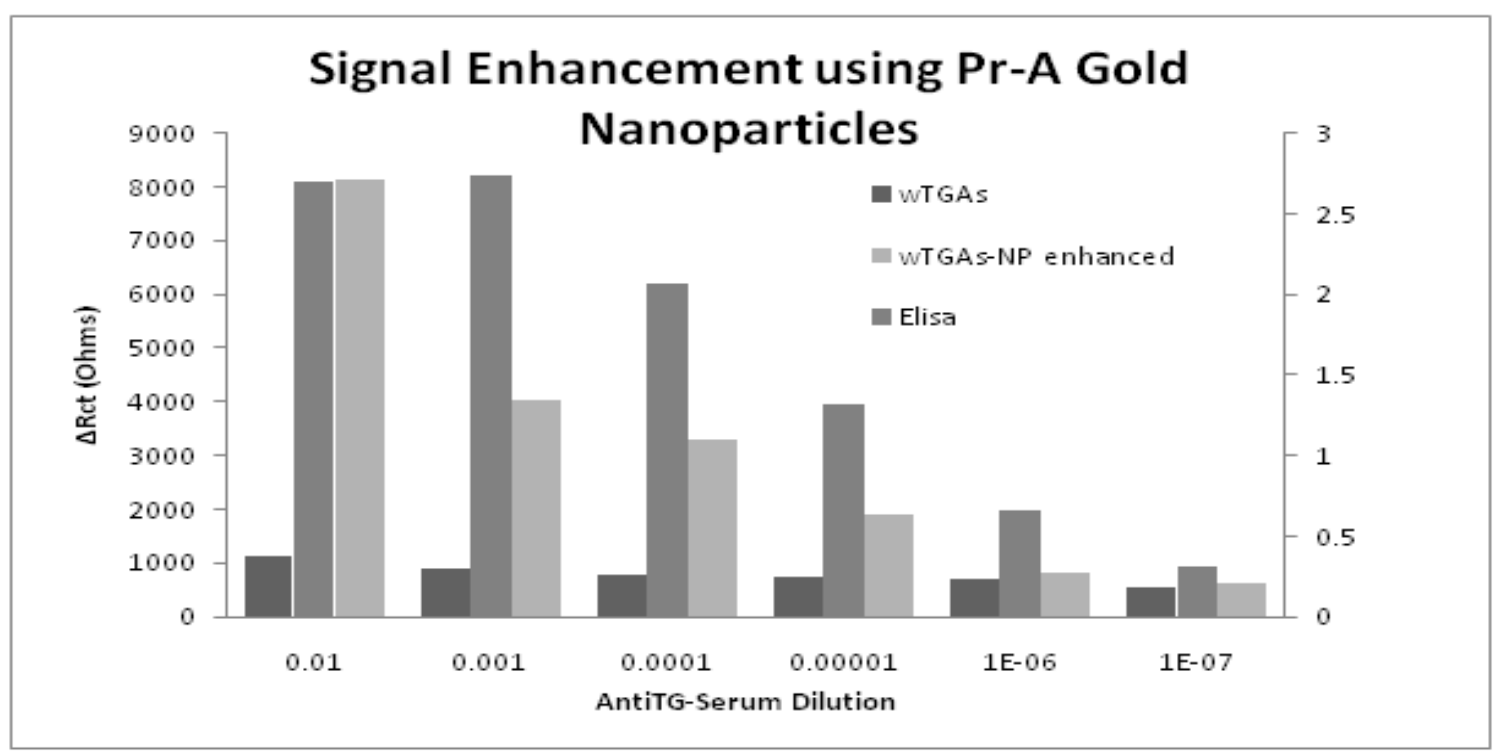

Figure 3.18: Histogram comparing three different detection methods. 


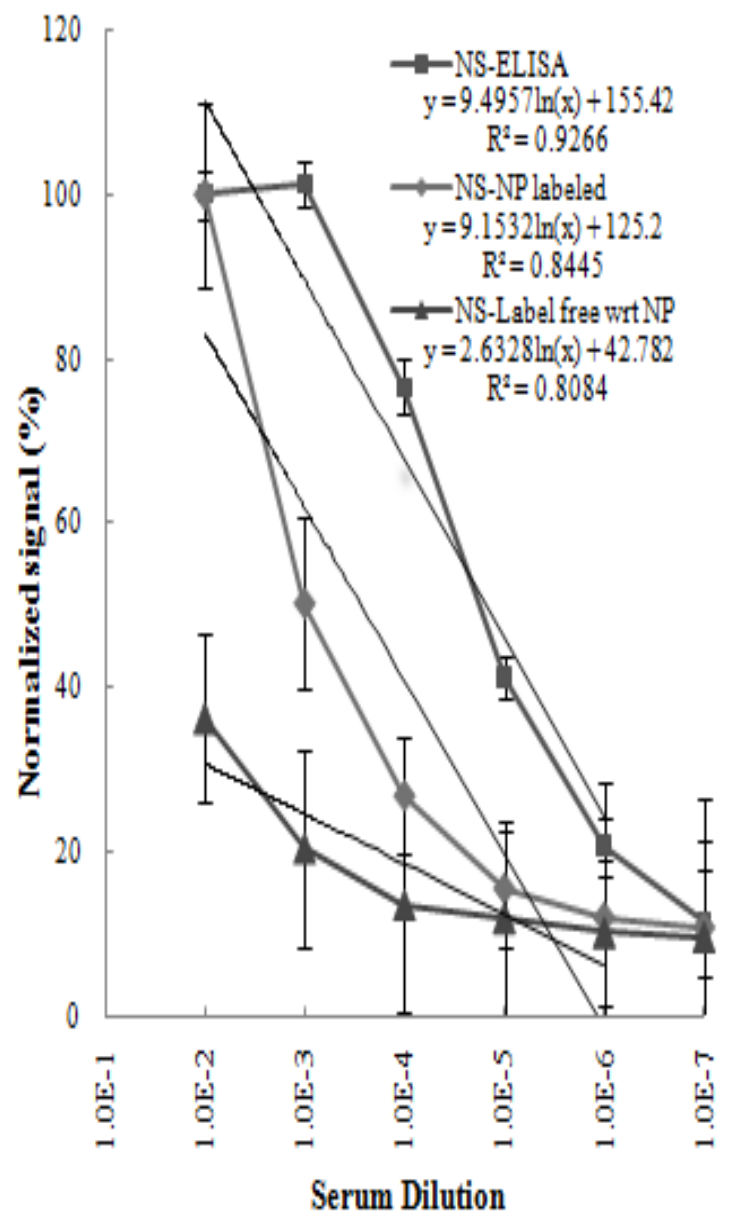

Figure 3.19: Plot showing the normalized signal strength of label free, Protein conjugated NPs, ELISA.

a wide range of analyte detection in a multiplexed format. 


\section{Chapter 4}

\section{Summary Conclusions}

Interdigitated electrodes were investigated in the current study as a possible transducers platform to act as a biosensor. In a simulated model,the nanogap IDEs were found to provide sufficiently high electric field and current density at applied potential, low enough to detect biomolecular activity without compromising the sensitivity of detection.

Electrochemical impedance spectroscopy was used as a detection technique and using EIS it was determined that the IDEs were quite sensitive to the electrochemical environment provided by change in concentration of redox probe molecules.

Self assembled monolayer (SAM) based functionalization chemistry was developed for immobilization of a protein antigen on the electrode surface, so that the antibodies in the sample can be captured from the applied samples. EIS was further used for detection of affinity based antibody capture on the electrodes. A suitable Randles equivalent circuit was used to analyze the physical changes occurring at the electrode surface due to immunoaffinity based biomolecular interactions.

It was found that the IDEA platform with EIS was capable of specific detection of biomolecules down to about $0.1 \mathrm{ng} / \mathrm{mL}$, An on-chip ELISA was performed as a traditional bioassay to validate the biosensor assay. However a comparison of label free

biosensor assay showed that the ELISA was at least 3.6 times more sensitive than the 
biosensor assay. This sensitivity gap was possibly due to the signal enhancing nature of the enzyme label in the ELISA. Gold nanoparticle were further used as suitable signal enhancing lables for EIS based IDEA sensor platform,

Protein-A gold nanoparticles were prepared and used as signal enhancing labels for the biosensor assay. The use of gold nanopartcles brought the sensitivity close to that of ELISA.

\subsubsection{Conclusion}

An electrochemical immunosensor based on IDEA's has been developed and compared with ELISA. The EIS results revealed that the SAM based surface modification technique allowed to capture more specific antibody on to the transducer surface for bioanalytical interaction with specific protein. The surface was also proved to be regenerative(the chip was reusable even after weeks of storage) with signal degradation of $\leq 10 \%$. The detection of label free $\alpha$-tTG was evaluated using EIS and the sensitivity achieved was in the range of nanograms per mL. This however was lower than the current gold standard ELISA. Introduction of labeled detection (nano particle conjugated protein) significantly increased the sensity of the sensor platform and the detection limits reached that of ELISA. This IDEA based immunosensor offers advantages of being highly sensitive, easy, and rapid to prepare (in batch manufacturing process), with a low assay time.

\subsubsection{Future work}

It has been proved that the IDEA is a suitable platform sensitive enough to challenge traditional bioassays and can be developed into a point of care device. Our lab is further focusing on development of bioassays protocols to reduce inter assay deviations, multiplexing the IDEA for simultaneous detection of several target analytes. Possible 
target analytes being pursued include biomarkesr for Cardiovascular disease, Cancer, allergies and autoimmune diseases and also environmental pollutants. Further other electrochemical techniques such as current amperometery and cyclic voltametery are also under investigation along with the EIS technique that has been used here. 


\section{References}

[1] B. Pejcic and R. D. Marco, "Impedance spectroscopy: Over 35 years of electrochemical sensor optimization." Electrochimica Acta., vol. 51(28), pp. 6217-6229, 2006

[2] B. D. Malhotra and A. Chaubey, "Biosensors for clinical diagnostics industry." Sensor Actuat B-Chem., vol. 91(1-3), pp. 117-127, 2003

[3] M. Abraham, W. Ehrfeld, V. Hessel, K. P. Kmper, M. Lacher and A. Picard, "Microsystem technology: Between research and industrial application." Microelectron Eng., vol 41, pp. 47-52, 1998

[4] B. van der Schoot, M. Boillat and N de Rooij, "Micro-instruments for life science research", IEEE Trans. Instrum Meas., vol. 50 (6), pp. 15381542,2001

[5] H. Nakamura and I. Karube, "Current research activity in biosensors" Anal Bioanal Chem., vol. 377, pp. 446468, 2003

[6] T. Vo-Dinh and B. Cullum, "Biosensors and biochips: advances in biological and medical diagnostics." Fresenius J Anal Chem., vol. 366 (6-7), pp. $540-51,2000$ 
[7] D. R. Thevenot, K. Toth, R. A. Durst and G. S. Wilson, "Electrochemical Biosensors: Recommended Definitions and Classification." Pure Appl Chem., vol. 71 (12), pp. 2333-2348, 1999

[8] L. Nicu, and T. Lechl, "Biosensors and tools for surface functionalization from the macro to the nanoscale: The way forward." J. Appl. Phys., vol. 104, pp. 111101, 2008

[9] S. P. Mohanty and E. Kougianos, "Biosensors: a tutorial" IEEE Potentials., vol. 25 (2), pp. 35-40, 2006

[10] J. S. Daniels and N. Pourmand, "Label-free impedance biosensors : Opportunities and challenges," Electroanal, vol. 19 (12), pp. 1239-1257, 2007

[11] Z. Ziqiang, Z. Jian and Z. Jianzhong, "An Overview of Si-based Biosensors." Sensor Letters, vol. 3 (2), pp. 71-88, 2005

[12] A.P.F. Turner, I. Karube and G. S. Wilson, "Biosensors: Fundamentals and Applications", eds., Oxford University Press,USA. 1990

[13] M. S. Belluzo, M. l. Ribone and C. M. Lagier, "Assembling Amperometric Biosensors for Clinical Diagnostics," Sensors, vol. 8 (3), pp. 13661399,2007

[14] A. Sharma and K. R. Rogers, "Biosensors" Meas.Sci.Technol., vol. 5, pp. 461,1994

[15] N. J. Renault, and S. V. Dzyadevych, "Conductometric Microbiosensors for Environmental Monitoring." Sensors, vol. 8(4), pp. 2569-2588, 2008 
[16] N. F. Sheppard Jr., R. C. Tucker and C. Wu, "Electrical conductivity measurements using microfabricated interdigitated electrodes," Anal. Chem., vol. 65 (9), pp. 1199-1202, 1993

[17] C.M.A. Brett and A.M. Oliveira-Brett, "Electrochemistry: Principles, Methods and Applications", eds., Oxford University Press, Oxford, UK, 1993

[18] M. I. Prodromidis, "Impedimetric immunosensors-A review." Electrochim Acta., vol. 55 (14), pp. 4227-4233, 2010

[19] M. Varshney and Y. Li, "Interdigitated array microelectrodes based impedance biosensors for detection of bacterial cells." Biosens Bioelectron., Vol 24 (10), pp. 2951-2960, 2009

[20] K.V. Singh, A. M. Whited, Y. Ragineni, T. W. Barrett, J. King and R. Solanki, "3D nanogap interdigitated electrode array biosensors," Anal bioanal chem. vol. 397(4), 1493-1502, 2010

[21] X. Huang, D. Nguyen, D.W. Greve, and M. M. Domach, "Simulation of Microelectrode Impedance Changes Due to Cell Growth." IEEE Sens. J., vol. 4 (5), pp. 576-583, 2004

[22] P. Van Gerwen, W. Laureys, G. Huyberechts, M. De Baeck, K. Baert, J. Suis, A. Varlan, W. Sansen, L. Hermans, and R. Mertens, "Nanoscaled interdigitated electrode arrays for biochemical sensors" In: International Conference on Transducers '97 Chicago., Solid State Sensors and Actuators, vol. 2., pp.907-910,1997 
[23] E. Barsoukov and J.R. Macdonald (Eds.), "Impedance Spectroscopy, Theory, Experiment, and Aplications," 2nd Edition, Wiley-Interscience, A John Wiley Sons, Inc., New Jersey, USA, 2005

[24] R. Ehret, W. Baumann, M. Brischwein, A. Schwinde, K. Stegbauer and B. Wolf, "Monitoring of cellular behaviour by impedance measurements on interdigitated electrode structures." Biosen Bioelectron., vol. 12 (1), pp. 29-41, 1997.

[25] Z.B. Stoynov, B.M. Grafov, B.S. Savova-Stoynova, V.V.Elkin, "Electrochemical Impedance," Nauka, Moscow, 1991.

[26] E. Katz and I. Willner, "Probing Biomolecular Interactions at Conductive and Semiconductive Surfaces by Impedance Spectroscopy: Routes to Impedimetric Immunosensors, DNA-Sensors, and Enzyme Biosensors," Electroanal, vol. 15(11), pp. 913-947, 2003

[27] A.J. Brad and L.R. Faulkner, "Electrochemical Methods: Fundamentals and Applications," Wiley, New York, 1980.

[28] 28. N. K. Chaki and K. Vijayamohanan, "Self-assembled monolayers as a tunable platform for biosensor applications." Biosens. Bioelectron., vol.17(1-2), pp. 1-12, 2002.

[29] S. K. Arya, P.R. Solanki, M. Datta and B. D. Malhotra, "Recent advances in self-assembled monolayers based biomolecular electronic devices.” Biosens. Bioelectron., vol. 24(9), 2810-2817, 2009

[30] Y. Huang, M. C. Bell, I. I.Suni, "Impedance biosensor for peanut protein Ara h 1.” Anal Chem., vol. 80(23), pp. 9157-61, 2008 